\title{
On Well-Structured Convex-Concave Saddle Point Problems and Variational Inequalities with Monotone Operators
}

\author{
Anatoli Juditsky* $\quad$ Arkadi Nemirovski ${ }^{\dagger}$
}

\begin{abstract}
For those acquainted with CVx (aka disciplined convex programming) of M. Grant and S. Boyd [9], the motivation of this work is the desire to extend the scope of CVX beyond convex minimization-to convex-concave saddle point problems and variational inequalities with monotone operators. To attain this goal, given a family $\mathcal{K}$ of cones (e.g., Lorentz, semidefinite, geometric, etc), we introduce the notions of $\mathcal{K}$-conic representation of a convex-concave saddle point problem and of variational inequality with monotone operator. We demonstrate that given such a representation of the problem of interest, the latter can be reduced straightforwardly to a conic problem on a cone from $\mathcal{K}$ and thus can be solved by (any) solver capable to handle conic problems on cones from $\mathcal{K}$ (e.g., Mosek or SDPT3 in the case of semidefinite cones). We also show that $\mathcal{K}$-representations of convex-concave functions and monotone vector fields admit a fully algorithmic calculus which helps to recognize the cases when a saddle point problem or variational inequality can be converted into a conic problem on a cone from $\mathcal{K}$ and to carry out such conversion.
\end{abstract}

\section{Introduction}

Along with emergence of powerful computational tools, one of important components of what is sometimes referred to as Interior-Point Revolution in Convex Optimization was the rise of (informal) notion of a "wellstructured convex problem" - convex optimization problem which can be reformulated as a conic problem on a cone from a "nice" family $\mathcal{K}$, most notably, the families of finite direct products of nonnegative rays (Linear Programming), Lorentz cones (Second Order Conic Programming), and semidefinite cones (Semidefinite Programming). Problems of the latter "magic families" cover basically all needs arising in applications of Convex Optimization to decision making, engineering, statistics, etc. At the same time, reformulating problem of interest as LP/SOCP/SDP makes the problem amenable for numerical processing by a "universal" SDP solver, so that end user should not care much about number-crunching. Today, there exist powerful universal SDP solvers such as SDPT3 [19] or industrial-grade Mosek 2], with permanently improving performance and reliability.

The "maiden form" of an optimization problem arising in applications usually is not the "nice conic" one, even when at the end of the day the problem can be cast into, say, an SDP program. Such transformation is carried out with the help of a special "calculus" (see, e.g., 3, 4, 5]) which allows to recognize that the problem at hand can be reformulated as, say, an SDP. This calculus, as any other, has two components: (a) "raw materials" - a collection of sets and functions possessing explicit representations via SDP (what exactly "representation" means, will be explained in Section 2), and (b) "calculus rules" expressing SDP representation of the result of a specific operation with functions/sets via SDP representations of the operands. It turns out that calculus rules for SDP representations cover all basic convexity-preserving operations with functions (like taking linear combinations with nonnegative coefficients and convexity-preserving compositions) and sets (like taking finite intersections and images/inverse images under affine mappings). Moreover, the calculus rules happen to be fully algorithmic and thus can be implemented on a compiler. This possibility is

\footnotetext{
*LJK, Université Grenoble Alpes, 700 Avenue Centrale, 38401 Domaine Universitaire de Saint-Martin-d'Hères, France, anatoli.juditsky@univ-grenoble-alpes.fr

${ }^{\dagger}$ ISyE, Georgia Institute of Technology, Atlanta, Georgia 30332, USA, nemirovs@isye.gatech.edu.
} 
utilized, for instance, in the CVX software of M. Grant and S. Boyd [9] which is the second-to-none in terms of its scope and user-friendliness tool for numerical processing of convex optimization problems.

So far, we were speaking about (constrained) convex minimization, and this is the usual scope of the calculus of well-structured convex problems. For example, given a number of SDP-representable functions, the calculus allows to obtain SDP representation of their maximum and thus convert to SDP program, in an automated fashion, the problem of minimizing this maximum over a feasible set given by an SDP representation. However, such calculus does not allow to get an SDP representation of the maximum of infinite family of SDP-representable functions, even when this family is perfectly well organized. As a result, convex-concave saddle point problems, well-structured from the "viewpoint of a human," cannot be "fed to CVX" as they are, and reducing such a problem to the conic form amenable for the existing powerful convex optimization software requires ad hoc work on case-to-case basis. Similar difficulties arise when instead of convex-concave saddle point problems someone is looking to solve numerically other problems with convex structure, most notably, variational inequalities (VI) with monotone operators. Monotone VI's can be thought of as the most general problems with convex structure: on one hand, other problems of this type (constrained convex minimization, convex-concave saddle points, convex Nash equilibrium problems) under extremely mild boundedness and regularity assumptions can be reduced to monotone VI's (for details, see e.g., [5, Section 5.6]). On the other hand, efficient black box oriented algorithms of convex minimization, like First Order or Ellipsoid methods, can be extended to monotone VI's (cf., e.g., 14]) without harming their efficiency estimates. There exists also rich literature on extending polynomial time Interior-Point algorithms onto linear and monotone complementarity problems, see, e.g., [11, 12, 7, 16, 17, 18, and references therein. In particular, it is well known that VI with affine monotone operator and domain admitting conic representation can be straightforwardly reduced to a conic optimization problem. In [13, Section 7.4] this fact was somehow extended to nonlinear monotone VI's from certain restricted class - those admitting an explicit "convex representation" allowing to reduce the VI of interest to an affine monotone VI on a properly modified domain; that reference develops also a rudimentary calculus of convex representations of monotone VI's. Some result:1 1 on reformulations of VI's as convex optimization problems can be found in 11. That being said, to the best of our knowledge, beyond the classes of VI's with affine monotone operators and related bilinear saddle point problems there is no much understanding of what is a "well structured" convex-concave saddle point problem or "genuine" — not potential - monotone VI, and of how such a problem should be represented in order for the representation to admit a meaningful algorithmic calculus, on one hand, and to allow for straightforward conversion of the problem into a nice conic program, say, SDP one, on the other.

The goal of what follows is to introduce the notion of "conic representations" of problems with convex structure, specifically, convex-concave saddle point problems and monotone VI's, via a given family $\mathcal{K}$ of cones (e.g., direct products of nonnegative rays/Lorentz/Semidefinite cones). As we will see, given such a representation, the problem of interest can be straightforwardly converted to a conic problem on a cone from $\mathcal{K}$, and representations in question admit a fully algorithmic calculus, similar to the calculus of conic representations of convex functions/sets. In Section 3 we develop conic representations of convex-concave functions, and then in Section 4 extend this framework to representations of monotone vector fields. Derivations in Section 4 can be viewed as a "well-structured" (i.e., conic representation-oriented) version of convex representation of a monotone operator introduced in [13, Section 7.4].

\section{Preliminaries}

Consider a family $\mathcal{K}$ of regular (i.e., closed, convex, pointed, and with nonempty interior) cones in Euclidean spaces such that the family

1. contains nonnegative ray,

2. is closed w.r.t. taking finite direct products of its members, and

3. is closed w.r.t. passing from a cone to its dual.

\footnotetext{
${ }^{1}$ dealing with the search for strong solutions to VI's with not necessarily monotone operator $F$ under rather restrictive assumptions that $x^{T} F(x)$ and $-F(x)$ are convex on polyhedral set $\mathcal{X} \subset \mathbf{R}_{+}^{n}$
} 
From now on we adopt the following convention: we operate with once for ever fixed family $\mathcal{K}$ of cones satisfying Assumptions 1-3, and whenever in the sequel we consider a cone, say $\mathbf{K}$, this cone is assumed to belong to $\mathcal{K}$. Besides this, $\mathbf{K}^{*}$ stands for the cone dual to $\mathbf{K}$.

- Given a cone $\mathbf{K} \in \mathcal{K}$ we call a constraint in variables $x \in \mathbf{R}^{n}$ of the form

$$
A x \leq_{\mathbf{K}} a
$$

$\mathcal{K}$-conic; as usual, $a \leq_{\mathbf{K}} b$ means that $b-a \in \mathbf{K}$. Note that a system

$$
A_{k} x \leq_{\mathbf{K}_{k}} a_{k}, 1 \leq k \leq K
$$

of finitely many $\mathcal{K}$-conic constraints is equivalent to a single $\mathcal{K}$-conic constraint 2

$$
\left[A_{1} ; \ldots ; A_{K}\right] x \leq_{\mathbf{K}}\left[a_{1} ; \ldots ; a_{K}\right]
$$

where $\mathbf{K}=\mathbf{K}_{1} \times \ldots \times \mathbf{K}_{K}$ belongs to $\mathcal{K}$ along with $\mathbf{K}_{k}, k \leq K$, since $\mathcal{K}$ is closed w.r.t. taking direct products. For similar reasons, augmenting a $\mathcal{K}$-conic constraint $A x \leq_{\mathbf{K}} a$ in variables $x$ by a system $S$ of finitely many linear equalities and nonstrict linear equalities, we get a conic constraint on $x$. Indeed, $S$ can be written equivalently as $B x \leq b$, so that the augmented constraint reads

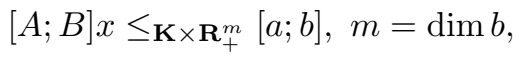

and the cone $\mathbf{K} \times \mathbf{R}_{+}^{m}$ belongs to $\mathcal{K}$ since the latter family contains $\mathbf{K}, \mathbf{R}_{+}$and is closed w.r.t. taking finite direct products.

- We call conic constraint (11) essentially strictly feasible, if $\mathbf{K}$ can be decomposed as $\mathbf{K}=\mathbf{R}_{+}^{m} \times \mathbf{L}$ with regular cone $\mathbf{L}$, so that (1) is of the form

$$
[P ; Q] x \leq_{\mathbf{R}_{+}^{m} \times \mathbf{L}} \leq[p ; q],
$$

and there exists $\bar{x}$ such that $P \bar{x} \leq p$ and $Q \bar{x}<_{\mathbf{L}} q$, the latter meaning that $q-Q \bar{x} \in \operatorname{int} \mathbf{L}$.

- We say that a set $X \subset \mathbf{R}^{n}$ is $\mathcal{K}$-representable if for properly selected $A, B, c$ and $\mathbf{K} \in \mathcal{F}$ one has

$$
X=\left\{x: \exists u: A x+B u \leq_{\mathbf{K}} c\right\} ;
$$

whenever this is the case, the $\mathcal{K}$-conic constraint $A x+B u \leq_{\mathbf{K}} c$ in the right hand side is called $\mathcal{K}$-representation of $X$. We call a function $f: \mathbf{R}^{n} \rightarrow \mathbf{R} \cup\{+\infty\} \mathcal{K}$-representable if the epigraph $\operatorname{Epi}\{f\}=\{(x, t): t \geq f(x)\}$ is $\mathcal{K}$-representable and we refer to a $\mathcal{K}$-representation of Epi $\{f\}$ as the $\mathcal{K}$ representation of $f$. In other words, asserting that $\mathcal{K}$-conic constraint $A x+t b+B u \leq_{\mathbf{K}} c$ represents $f$ is the same as saying that the following equivalence takes place:

$$
t \geq f(x) \Leftrightarrow \exists u: A x+t b+B u \leq_{\mathbf{K}} c .
$$

Observe that the level sets $\{x: f(x) \leq a\}, a \in \mathbf{R}$, of $\mathcal{K}$-representable function $f$ are $\mathcal{K}$-representable:

$$
\left\{t \geq f(x) \Leftrightarrow \exists u: A x+t b+B u \leq_{\mathbf{K}} c\right\} \Rightarrow\{x: f(x) \leq a\}=\left\{x: \exists u: A x+B y \leq_{\mathbf{K}} c-a b\right\} .
$$

Thus, given $\mathcal{K}$-representations $\left\{x: \exists u: A x+B u \leq_{\mathbf{K}} c\right\}$ of $X \subset \mathbf{R}^{n}$ and $\left\{t \geq f(x) \Leftrightarrow \exists v: C x+t d+E v \leq_{\mathbf{L}} g\right\}$ of $f: \mathbf{R}^{n} \rightarrow \mathbf{R} \cup\{+\infty\}$, the problem

$$
\min _{x \in X} f(x)
$$

is equivalent to the conic problem

$$
\min _{x, t, u, v}\left\{t:[A x+B u ; C x+t d+E v] \leq_{\mathbf{K} \times \mathbf{L}}[c ; g]\right\}
$$

\footnotetext{
${ }^{2}$ We follow the "Matlab convention" for matrices: $[A, B]$ and $[A ; B]$ denote, respectively, the horizontal and vertical concatenations of two matrices of compatible dimensions.
} 
on a cone from $\mathcal{K}$, where equivalence of $(\mathcal{P})$ and $(\mathcal{Q})$ precisely means that $x$ is a feasible solution to $(\mathcal{P})$ with a finite value of the objective if and only if there exist $t, u, v$ such that $t \geq f(x)$ and $x, t, u, v$ is feasible for $(\mathcal{Q})$.

$\mathcal{K}$-representable functions/sets admit fully algorithmic calculus: all basic convexity-preserving operations with sets and functions, (e.g., taking finite intersections, images/inverse images under affine mappings, and direct products of sets, or taking linear combinations with nonnegative coefficients and convexity-preserving compositions of functions) as applied to $\mathcal{K}$-representable operands, produce $\mathcal{K}$-representable results, with representations of the results readily given by those of the operands; for details, see, e.g., 44, 5. When a solver for conic problems on cones from $\mathcal{K}$ is available, this calculus allows one to recognize that in the problem $(\mathcal{P})$ of interest objective $f$ and domain $X$ are $\mathcal{K}$-representable and find the corresponding $\mathcal{K}$-representations 3 so the problem of interest can be converted into a conic problem $(\mathcal{Q})$ on a cone from $\mathcal{K}$ and solved by the solver at hand.

From now on, we operate with a once for ever fixed family $\mathcal{K}$ of cones satisfying the above Assumptions 1-3, which allows for the convention as follows: whenever in the sequel a cone arises, this cone, if the otherwise is explicitly stated, belongs to $\mathcal{K}$. Besides this, $\mathbf{K}^{*}$ stands for the cone dual to $\mathbf{K}$.

\section{Well-structured convex-concave saddle point problems}

As far as its paradigm and set of rules are concerned, "calculus of $\mathcal{K}$-representability" for, say, $\mathcal{K}$ specified as $\mathcal{S D} \mathcal{P}$ (finite direct products of semidefinite cones) covers all "basic" needs of "well-structured" convex minimization. There is, however, an exception - the case in which the objective in the convex problem of interest

$$
\min _{x \in \mathcal{X}} \bar{\Psi}(x)
$$

is given implicitly:

$$
\bar{\Psi}(x)=\sup _{y \in \mathcal{Y}} \psi(x, y)
$$

where $\mathcal{Y}$ is convex set and $\psi: \mathcal{X} \times \mathcal{Y} \rightarrow \mathbf{R}$ is convex-concave (i.e., convex in $x \in \mathcal{X}$ and concave in $y \in \mathcal{Y}$ ) and continuous. Problem $(P)$ with objective given by (2) is called "primal problem associated with the convex-concave saddle point problem $\min _{x \in \mathcal{X}} \max _{y \in \mathcal{Y}} \psi(x, y)$," and problems of this type do arise in some applications of well-structured convex optimization. We are about to define a saddle point version of $\mathcal{K}$-representability along with the corresponding calculus which allows to convert "K $\mathcal{K}$-representable convexconcave saddle point problems" into usual conic problems on cones from $\mathcal{K}$.

\subsection{Conic representability of convex-concave function-definition}

Let $\mathcal{X}, \mathcal{Y}$ be nonempty convex sets given by $\mathcal{K}$-representations:

$$
\mathcal{X}=\left\{x: \exists \xi: A x+B \xi \leq_{\mathbf{K}_{\mathcal{X}}} c\right\}, \mathcal{Y}=\left\{y: \exists \eta: C y+D \eta \leq_{\mathbf{K}_{\mathcal{Y}}} e\right\} \quad\left[\mathbf{K}_{\mathcal{X}} \in \mathcal{K}, \mathbf{K}_{\mathcal{Y}} \in \mathcal{K}\right]
$$

Let us say that a convex-concave continuous function $\psi(x, y): \mathcal{X} \times \mathcal{Y} \rightarrow \mathbf{R}$ is $\mathcal{K}$-representable on $\mathcal{X} \times \mathcal{Y}$, if it admits representation of the form

$$
\forall(x \in \mathcal{X}, y \in \mathcal{Y}): \psi(x, y)=\inf _{f, t, u}\left\{f^{T} y+t: P f+t p+Q u+R x \leq_{\mathbf{K}} s\right\}
$$

where $\mathbf{K} \in \mathcal{K}$. We call representation (3) essentially strictly feasible, if the conic constraint

$$
P f+t p+Q u \leq_{\mathbf{K}} s-R x
$$

in variables $f, t, u$ is essentially strictly feasible for every $x \in \mathcal{X}$.

\footnotetext{
${ }^{3}$ Note that calculus rules are fully algorithmic and thus can be implemented by a compiler, the most famous example being the CVX software [9] which can handle "semidefinite representability" (family $\mathcal{K}$ comprised of direct products of semidefinite cones), and as a byproduct - conic quadratic representability.
} 


\subsection{Main observation}

Assume that $\mathcal{Y}$ is compact and is given by essentially strictly feasible $\mathcal{K}$-representation

$$
\mathcal{Y}=\left\{y: \exists \eta: C y+D \eta \leq_{\mathbf{K}_{\mathcal{Y}}} e\right\} .
$$

Then problem $(P)$ can be processed as follows: for $x \in \mathcal{X}$ we have

$$
\begin{aligned}
\bar{\Psi}(x) & =\max _{y \in \mathcal{Y}} \inf _{f, t, u}\left[f^{T} y+t: P f+t p+Q u+R x \leq_{\mathbf{K}} s\right] \\
& =\inf _{f, t, u}\left\{\max _{y \in \mathcal{Y}}\left[f^{T} y+t\right]: P f+t p+Q u+R x \leq_{\mathbf{K}} s\right\} \quad\left[\begin{array}{l}
\text { Sion-Kakutani Theorem; recall } \\
\text { that } \mathcal{Y} \text { is convex and compact }
\end{array}\right] \\
= & \inf _{f, t, u}\left\{\max _{y, \eta}\left[f^{T} y: C y+D \eta \leq_{\mathbf{K}_{\mathcal{Y}}} e\right]+t: P f+t p+Q u+R x \leq_{\mathbf{K}} s\right\} \\
= & \inf _{f, t, u}\left\{\min _{\lambda}\left[\lambda^{T} e: C^{T} \lambda=f, D^{T} \lambda=0, \lambda \geq_{\mathbf{K}_{\mathcal{Y}}^{*}} 0\right]+t: P f+t p+Q u+R x \leq_{\mathbf{K}} s\right\} \\
& {\left[\begin{array}{l}
\text { by strong conic duality, see, e.g., } \\
\text { recall that (44) is essentially strictly feasible, }
\end{array}\right.}
\end{aligned}
$$

so that the problem of interest

$$
\min _{x \in \mathcal{X}} \bar{\Psi}(x)
$$

reduces to the explicit $\mathcal{K}$-conic problem

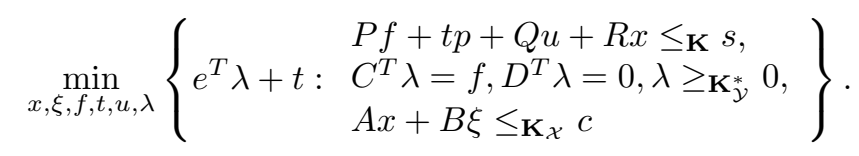

Here, "reduction" means that the $x$-component of a feasible solution $\zeta=(x, \xi, f, t, u, \lambda)$ to $(b)$ is a feasible solution to $(a)$ with the value of the objective of the latter problem at $x$ being $\leq$ the value of the objective of $(b)$ at $\zeta$, and the optimal values in $(a)$ and $(b)$ are the same. Thus, as far as building feasible approximate solutions of a prescribed accuracy $\epsilon>0$ in terms of the objective are concerned, problem $(a)$ reduces to the explicit conic problem $(b)$. Note, however, that $(a)$ and $(b)$ are not "exactly the same" - it may happen that $(a)$ is solvable while $(b)$ is not so. "For all practical purposes," this subtle difference is of no importance since in actual computations exactly optimal solutions usually are not reachable anyway.

Discussion. Note that for continuous convex-concave function $\psi: \mathcal{X} \times \mathcal{Y} \rightarrow \mathbf{R}$ the set

$$
\mathcal{Z}=\left\{[f ; t ; x]: x \in \mathcal{X}, f^{T} y+t \geq \psi(x, y) \forall y \in \mathcal{Y}\right\}
$$

clearly is convex, and by the standard Fenchel duality we have

$$
\forall(x \in \mathcal{X}, y \in \mathcal{Y}): \psi(x, y)=\inf _{f, t}\left[f^{T} y+t:[f ; t ; x] \in \mathcal{Z}\right]
$$

$\mathcal{K}$-representability of $\psi$ on $\mathcal{X} \times \mathcal{Y}$ means that (5) is preserved when replacing the set $\mathcal{Z}$ with its properly selected $\mathcal{K}$-representable subset. Given that $\mathcal{Z}$ is convex, this assumption seems to be not too restrictive; taken together with $\mathcal{K}$-representability of $\mathcal{X}$ and $\mathcal{Y}$, it can be treated as the definition of $\mathcal{K}$-representability of the convex-concave function $\psi$. The above derivation shows that convex-concave saddle point problem with $\mathcal{K}$-representable domain and cost function (more precisely, the primal minimization problem $(P)$ induced by this saddle point problem) can be represented in explicit $\mathcal{K}$-conic form, at least when the $\mathcal{K}$-representations of the cost and of (compact) $\mathcal{Y}$ are essentially strictly feasible.

Note also that if $\mathcal{X}$ and $\mathcal{Y}$ are convex sets and a function $\psi(x, y): \mathcal{X} \times \mathcal{Y} \rightarrow \mathbf{R}$ admits representation (3), then $\psi$ automatically is convex in $x \in \mathcal{X}$ and concave in $y \in \mathcal{Y}$. 


\subsection{Symmetry}

Assume that representation (3) is essentially strictly feasible. Then for all $x \in \mathcal{X}, y \in \mathcal{Y}$ we have by conic duality

$$
\begin{aligned}
\psi(x, y) & =\inf _{f, t, u}\left\{f^{T} y+t: P f+t p+Q u+R x \leq_{\mathbf{K}} s\right\} \\
& =\sup _{\bar{u} \in \mathbf{K}^{*}}\left\{\bar{u}^{T}[R x-s]: P^{T} \bar{u}+y=0, p^{T} \bar{u}+1=0, Q^{T} \bar{u}=0\right\},
\end{aligned}
$$

whence, setting

$$
\overline{\mathcal{X}}=\mathcal{Y}, \overline{\mathcal{Y}}=\mathcal{X}, \bar{x}=y, \bar{y}=x, \bar{\psi}(\bar{x}, \bar{y})=-\psi(\bar{y}, \bar{x})=-\psi(x, y)
$$

we have

$$
\begin{aligned}
& (\forall \bar{x} \in \overline{\mathcal{X}}, \bar{y} \in \overline{\mathcal{Y}}): \\
& \begin{aligned}
\bar{\psi}(\bar{x}, \bar{y}) & =-\psi(x, y)=\inf _{\bar{u} \in \mathbf{K}^{*}}\left\{-\bar{u}^{T}[R x-s]: P^{T} \bar{u}+y=0, p^{T} \bar{u}+1=0, Q^{T} \bar{u}=0\right\} \\
= & \inf _{\bar{f}, \bar{t}, \bar{u}}\{\bar{f}^{T} \bar{y}+\bar{t}: \underbrace{\left[\begin{array}{l}
\bar{f}=-R^{T} \bar{u}, \bar{t}=s^{T} \bar{u}, Q^{T} \bar{u}=0, \\
p^{T} \bar{u}+1=0, P^{T} \bar{u}+\bar{x}=0, \bar{u} \in \mathbf{K}^{*}
\end{array}\right]}_{\Leftrightarrow \bar{P} \bar{f}+\bar{t} \bar{p}+\bar{Q} \bar{u}+\bar{R} \bar{x} \leq \overline{\mathbf{K}}^{\bar{s}}}\}
\end{aligned}
\end{aligned}
$$

with $\overline{\mathbf{K}} \in \mathcal{K}$. We see that a (essentially strictly feasible) $\mathcal{K}$-representation of convex-concave function $\psi$ on $\mathcal{X} \times \mathcal{Y}$ induces straightforwardly a $\mathcal{K}$-representation of the "symmetric entity" - the convex-concave function $\bar{\psi}(y, x)=-\psi(x, y)$ on $\mathcal{Y} \times \mathcal{X}$, with immediate consequences for converting the optimization problem

$$
\sup _{y \in \mathcal{Y}}\left[\underline{\Psi}(y):=\inf _{x \in \mathcal{X}} \psi(x, y)\right]
$$

into the standard conic form.

\subsection{Calculus of conic representations of convex-concave functions}

Representations of the form (3) admit a calculus.

\subsubsection{Raw materials}

Raw materials for the calculus are given by

1. Functions $\psi(x, y)=a(x)$, where $a(x)$, Dom $a \supset \mathcal{X}$, is $\mathcal{K}$-representable:

$$
t \geq a(x) \Leftrightarrow \exists u: \bar{R} x+t \bar{p}+\bar{Q} u \leq \overline{\mathbf{K}} \bar{s}
$$

In this case

$$
\psi(x, y)=\inf _{f, t, u}\{f^{T} y+t: \underbrace{f=0, \bar{R} x+t \bar{p}+\bar{Q} u \leq \overline{\mathbf{K}} \bar{s}}_{\Leftrightarrow P f+t p+Q u+R x \leq \mathbf{K} s \text { with } \mathbf{K} \in \mathcal{K}}\} .
$$

2. Functions $\psi(x, y)=-b(y)$, where $b(y)$, Dom $b \supset \mathcal{Y}$, is $\mathcal{K}$-representable:

$$
t \geq b(y) \Leftrightarrow \exists u: \bar{R} y+t \bar{p}+\bar{Q} u \leq_{\overline{\mathbf{K}}} \bar{s}
$$

with essentially strictly feasible $\mathcal{K}$-representation. In this case

$$
\begin{aligned}
\psi(x, y) & =-b(y)=-\inf _{t, u}\{t: \bar{R} y+t \bar{p}+\bar{Q} u \leq \overline{\mathbf{K}} \bar{s}\} \\
& =-\sup _{u \in \overline{\mathbf{K}}^{*}}\left\{-\left[\bar{R}^{T} u\right]^{T} y-s^{T} u:-u^{T} \bar{p}=1, \bar{Q}^{T} u=0\right\} \quad \text { [by conic duality] } \\
& =\inf _{f, t, u}\{f^{T} y+t: \underbrace{f=R^{T} u, t=s^{T} u, \bar{p}^{T} u+1=0, \bar{Q}^{T} u=0, u \geq \overline{\mathbf{K}}^{*} 0}_{\Leftrightarrow P f+t p+Q u \leq \mathbf{K} s \text { with } \mathbf{K} \in \mathcal{K}}\} .
\end{aligned}
$$


3. Bilinear functions:

$$
\psi(x, y) \equiv a^{T} x+b^{T} y+x^{T} A y+c \Rightarrow \psi(x, y)=\min _{f, t}\{f^{T} y+t: \underbrace{f=A^{T} x+b, t=a^{T} x+c}_{\Leftrightarrow P f+t p+R x \leq s}\} .
$$

4. "Generalized bilinear functions." Let $\mathbf{U} \in \mathcal{K}$ and $E$ be the embedding Euclidean space of $\mathbf{U}$.

(a) Let $\overline{\mathcal{X}}$ be a nonempty $\mathcal{K}$-representable set, and let continuous mapping $F(x): \overline{\mathcal{X}} \rightarrow E$ possess $\mathcal{K}$-representable $\mathbf{U}$-epigraph 4

$$
\mathrm{Epi}_{\mathbf{U}} F:=\left\{(x, z) \in \overline{\mathcal{X}} \times E, z \geq_{\mathbf{U}} F(x)\right\}=\{(x, z): \exists u: \bar{R} x+\bar{S} z+\bar{T} u \leq \overline{\mathbf{K}} \bar{s}\} .
$$

Then the function

$$
\bar{\psi}(x, y)=y^{T} F(x): \overline{\mathcal{X}} \times \mathbf{U}^{*} \rightarrow \mathbf{R}
$$

is $\mathcal{K}$-representable on $\overline{\mathcal{X}} \times \mathbf{U}^{*}$ :

$$
\begin{aligned}
& \forall\left(x \in \overline{\mathcal{X}}, y \in \mathbf{U}^{*}\right): \\
& \begin{aligned}
\bar{\psi}(x, y) & =y^{T} F(x)=\inf _{f}\left\{f^{T} y: f \geq \mathbf{U} F(x)\right\} \\
& =\inf _{f, u}\left\{f^{T} y: \bar{R} x+\bar{S} z+\bar{T} u \leq \overline{\mathbf{K}} \bar{s}\right\}
\end{aligned}
\end{aligned}
$$

(b) Let $\mathcal{Y}$ be a nonempty $\mathcal{K}$-representable set, and let continuous mapping $G(y): \mathcal{Y} \rightarrow E$ possess $\mathcal{K}$-representable $\mathbf{U}^{*}$-hypograph,

$$
\begin{aligned}
\operatorname{Hypo}_{\mathbf{U}^{*}} G & :=\left\{(y, w) \in \mathcal{Y} \times E: w \leq \mathbf{U}^{*} G(y)\right\} \\
& =\{(y, w): \exists u: \underline{R} y+\underline{S} w+\underline{Q} u \geq \underline{\mathbf{K}} \underline{s}\} \quad[\underline{\mathbf{K}} \in \mathcal{K}],
\end{aligned}
$$

the representation being essentially strictly feasible. Then the function

$$
\underline{\psi}(\underline{x}, y)=\underline{x}^{T} G(y): \mathbf{U} \times \mathcal{Y} \rightarrow \mathbf{R}
$$

is $\mathcal{K}$-representable on $\mathbf{U} \times \mathcal{Y}$ :

$$
\begin{aligned}
& \forall(\underline{x} \in \mathbf{U}, y \in \mathcal{Y}): \\
& \begin{aligned}
\underline{\psi}(\underline{x}, y) & =\underline{x}^{T} G(y)=\sup _{w}\left\{\underline{x}^{T} w: w \leq \mathbf{U}^{*} G(y)\right\} \quad[\text { due to } \underline{x} \in \mathbf{U}] \\
& =\sup _{w, u}\left\{\underline{x}^{T} w: \underline{R} y+\underline{S} w+\underline{Q} u \geq \underline{\mathbf{K}} \underline{s}\right\} \\
& =\inf _{\lambda}\left\{[\underline{s}-\underline{R} y]^{T} \lambda: \underline{S}^{T} \lambda=\underline{x}, \underline{Q}^{T} \lambda=0, \lambda \in \underline{\mathbf{K}}^{*}\right\} \quad[\text { by conic duality] } \\
& =\inf _{f, t, u=[\lambda ; w]}\{f^{T} y+t: \underbrace{\left[\begin{array}{l}
f+\underline{R}^{T} \lambda=0, \underline{s}^{T} \lambda=t, \\
\underline{S}^{T} \lambda=\underline{x}, \underline{Q}^{T} \lambda=0, \lambda \in \underline{\mathbf{K}}^{*}
\end{array}\right]}_{\Leftrightarrow P f+t p+Q u+R \underline{x} \leq \mathbf{K}^{s}}\}[\mathbf{K} \in \mathcal{K}] .
\end{aligned}
\end{aligned}
$$

(c) Let $\mathcal{Y}$ and $G(\cdot)$ be as in item $4 \mathrm{~b}$ ) with $G(\mathcal{Y}) \subset \mathbf{U}^{*}$, let $\mathcal{X}$ be a nonempty $\mathcal{K}$-representable set, and let

$$
F(x): \mathcal{X} \rightarrow \mathbf{U}
$$

be continuous $\mathbf{U}$-convex mapping with $\mathcal{K}$-representable $\mathbf{U}$-epigraph:

$$
\begin{aligned}
\operatorname{Epi}_{\mathbf{U}} F & :=\{(x, z): x \in \mathcal{X}, z \geq \mathbf{U} F(x)\} \\
& =\left\{x, z: \exists v: \widehat{R} x+\widehat{S} z+\widehat{Q} v \leq_{\widehat{\mathbf{K}}} \widehat{s}\right\} \quad[\widehat{\mathbf{K}} \in \mathcal{K}]
\end{aligned}
$$

\footnotetext{
${ }^{4}$ This implies, in particular that the $\mathbf{U}$-epigraph of $F$ is convex, or, which is the same, that $F$ is $\mathbf{U}$-convex:$$
\forall\left(x^{\prime} ; x^{\prime \prime} \in \overline{\mathcal{X}}, \lambda \in[0,1]\right): F\left(\lambda x^{\prime}+(1-\lambda) x^{\prime \prime}\right) \leq_{\mathbf{U}} \lambda F\left(x^{\prime}\right)+(1-\lambda) F\left(x^{\prime \prime}\right) .
$$ 
Then the function

$$
\psi(x, y)=F^{T}(x) G(y): \mathcal{X} \times \mathcal{Y} \rightarrow \mathbf{R}
$$

is continuous convex-concave and admits $\mathcal{K}$-representation as follows:

$$
\begin{aligned}
& \forall(x \in \mathcal{X}, y \in \mathcal{Y}): \\
& \begin{aligned}
\psi(x, y) & =F^{T}(x) G(y) \\
& =\inf _{z}\left\{z^{T} G(y): z \geq \mathbf{U} F(x)\right\} \quad\left[\text { since } G(y) \in \mathbf{U}^{*}\right] \\
& =\inf _{z, v}\left\{z^{T} G(y): \widehat{R} x+\widehat{S} z+\widehat{Q} v \leq_{\widehat{\mathbf{K}}} \widehat{s}\right\}[\text { by (7) }] \\
& =\inf _{z, v}\left\{\inf _{f, t}\left\{f^{T} y+t: P f+t p+Q u+R z \leq_{\mathbf{K}} s\right\}: \widehat{R} x+\widehat{S} z+\widehat{Q} v \leq_{\widehat{\mathbf{K}}} \widehat{s}\right\}
\end{aligned}
\end{aligned}
$$

due to (6) - note that on the domain on which $\inf _{z, v}$ is taken we have $z \geq \mathbf{U} F(x) \in \mathbf{U}$, making (6) applicable. We conclude that

$$
\psi(x, y)=\inf _{f, t, u=[z ; v]}\{f^{T} y+t: \underbrace{\left[\begin{array}{l}
P f+t p+Q u+R z \leq_{\mathbf{K}} s, \\
\widehat{R} x+\widehat{S} z+\widehat{Q} v \leq_{\widehat{\mathbf{K}}} \widehat{s}
\end{array}\right]}_{\Leftrightarrow \widetilde{P} f+t \widetilde{p}+\widetilde{Q} u+\widetilde{R} x \leq_{\widetilde{\mathbf{K}}} \widetilde{c}}\}[\widetilde{\mathbf{K}} \in \mathcal{K}] .
$$

\subsubsection{Basic calculus rules}

Basic calculus rules are as follows.

1. [Direct summation] Let $\theta_{i}>0, i \leq I$, and let

$$
\begin{aligned}
& \forall\left(x^{i} \in \mathcal{X}^{i}, y^{i} \in \mathcal{Y}^{i}, i \leq I\right): \\
& \psi_{i}\left(x^{i}, y^{i}\right)=\inf _{f_{i}, t_{i}, u_{i}}\left\{f_{i}^{T} y^{i}+t_{i}: P_{i} f_{i}+t_{i} p_{i}+Q_{i} u_{i}+R_{i} x^{i} \leq_{\mathbf{K}_{i}} s_{i}\right\}
\end{aligned}
$$

Then

$$
\begin{aligned}
& \forall\left(x=\left[x^{1} ; \ldots ; x^{I}\right] \in \mathcal{X}=\mathcal{X}_{1} \times \ldots \times \mathcal{X}_{I}, y=\left[y^{1} ; \ldots ; y^{I}\right] \in \mathcal{Y}=\mathcal{Y}_{1} \times \ldots \times \mathcal{Y}_{I}\right): \\
& \psi(x, y):=\sum_{i} \theta_{i} \psi_{i}\left(x^{i}, y^{i}\right) \\
& =\inf _{f, t, u=\left\{f_{i}, t_{i}, u_{i}, i \leq I\right\}}\{f^{T} y+t: \underbrace{\begin{array}{l}
f=\left[\theta_{1} f_{1} ; \ldots ; \theta_{I} f_{I}\right], t=\sum_{i} \theta_{i} t_{i}, \\
P_{i} f_{i}+t_{i} p_{i}+Q_{i} u_{i}+R_{i} x^{i} \leq \mathbf{K}_{i}
\end{array} s_{i}, 1 \leq i \leq I}_{\Leftrightarrow P f+t p+Q u+R x \leq_{\mathbf{K}} s \text { with } \mathbf{K} \in \mathcal{K}}\}
\end{aligned}
$$

2. [Affine substitution of variables] Let

$$
\begin{aligned}
& \forall\left(\xi \in \mathcal{X}^{+}, \eta \in \mathcal{Y}^{+}\right): \\
& \psi_{+}(\xi, \eta)=\inf _{f_{+}, t_{+}, u_{+}}\left\{f_{+}^{T} \eta+t_{+}: P_{+} f_{+}+t_{+} p_{+}+Q_{+} u_{+}+R_{+} \xi \leq_{\mathbf{K}_{+}} s_{+}\right\}
\end{aligned}
$$

and

$$
x \mapsto A x+b: \mathcal{X} \rightarrow \mathcal{X}^{+}, y \mapsto B y+c: \mathcal{Y} \rightarrow \mathcal{Y}^{+}
$$

Then

$$
\begin{aligned}
& \forall(x \in \mathcal{X}, y \in \mathcal{Y}): \\
& \begin{aligned}
\psi(x, y):= & \psi_{+}(A x+b, B y+c) \\
& =\inf _{f_{+}, t_{+}, u_{+}}\left\{f_{+}^{T}(B y+c)+t_{+}: P_{+} f_{+}+t_{+} p_{+}+Q_{+} u_{+}+R_{+}[A x+b] \leq_{\mathbf{K}_{+}} s_{+}\right\} \\
& =\inf _{f, t, u=\left[f_{+} ; t_{+} ; u_{+}\right]}\{f^{T} y+t: \underbrace{\left[\begin{array}{l}
f=B^{T} f_{+}, t=t_{+}+f_{+}^{T} c \\
P_{+} f_{+}+t_{+} p_{+}+Q_{+} u_{+}+R_{+} A x \mathbf{K}_{+} s_{+}-R_{+} b
\end{array}\right]}_{\Leftrightarrow P f+t p+Q u+R x \leq_{\mathbf{K}} s \text { with } \mathbf{K} \in \mathcal{K}}\}
\end{aligned}
\end{aligned}
$$


3. [Taking conic combinations] This rule, evident by itself, is a combination of the two preceding rules: Let $\theta_{i}>0$ and $\psi_{i}(x, y): \mathcal{X} \times \mathcal{Y} \rightarrow \mathbf{R}, i \leq I$, be such that

$$
\begin{aligned}
& \forall(x \in \mathcal{X}, y \in \mathcal{Y}): \\
& \psi_{i}(x, y)=\inf _{f_{i}, t_{i}, u_{i}}\left\{f_{i}^{T} y^{i}+t_{i}: P_{i} f_{i}+t_{i} p_{i}+Q_{i} u_{i}+R_{i} x^{i} \leq_{\mathbf{K}_{i}} s_{i}\right\}
\end{aligned}
$$

Then

$$
\begin{aligned}
& \forall(x \in \mathcal{X}, y \in \mathcal{Y}): \\
& \psi(x, y):=\sum_{i} \theta_{i} \psi_{i}(x, y) \\
& =\inf _{f, t, u=\left\{f_{i}, t_{i}, u_{i}, i \leq I\right\}}\{f^{T} y+t: \underbrace{\left[\begin{array}{l}
f=\sum_{i} \theta_{i} f_{i}, t=\sum_{i} \theta_{i} t_{i}, \\
P_{i} f_{i}+t_{i} p_{i}+Q_{i} u_{i}+R_{i} x^{i} \leq \mathbf{K}_{i} s_{i}, 1 \leq i \leq I
\end{array}\right]}_{\Leftrightarrow P f+t p+Q u+R x \leq \mathbf{K}^{s} \text { with } \mathbf{K} \in \mathcal{K}} .
\end{aligned}
$$

4. [Projective transformation in $x$-variable] Let

$$
\forall(x \in \mathcal{X}, y \in \mathcal{Y}): \psi(x, y)=\inf _{f, t, u}\left\{f^{T} y+t: P f+t p+Q u+R x \leq_{\mathbf{K}} s\right\} .
$$

Then

$$
\begin{aligned}
& \left(\forall(\alpha, x): \alpha>0, \alpha^{-1} x \in \mathcal{X}, \forall y \in \mathcal{Y}\right) \\
& \bar{\psi}((\alpha, x), y):=\alpha \psi\left(\alpha^{-1} x, y\right)=\inf _{f, t, u}\left\{f^{T} y+t: P f+t p+Q u+R x-\alpha s \leq_{\mathbf{K}} 0\right\} .
\end{aligned}
$$

5. [Superposition in $x$-variable] Let $\mathcal{X}, \mathcal{Y}$ be $\mathcal{K}$-representable, $\overline{\mathcal{X}}$ be a $\mathcal{K}$-representable subset of some $\mathbf{R}^{n}$, and let $\mathcal{K} \ni \mathbf{U}$ be a cone in $\mathbf{R}^{n}$. Furthermore, assume that

$$
\bar{\psi}(\bar{x}, y): \overline{\mathcal{X}} \times \mathcal{Y} \rightarrow \mathbf{R}
$$

is a continuous convex-concave function which is $\mathbf{U}$-nondecreasing in $\bar{x} \in \overline{\mathcal{X}}$, i.e.

$$
\forall\left(y \in \mathcal{Y}, \bar{x}^{\prime}, \bar{x}^{\prime \prime} \in \overline{\mathcal{X}}: \bar{x}^{\prime} \leq_{\mathbf{U}} \bar{x}^{\prime \prime}\right): \bar{\psi}\left(\bar{x}^{\prime \prime}, y\right) \geq \bar{\psi}\left(\bar{x}^{\prime}, y\right),
$$

and admits $\mathcal{K}$-representation on $\overline{\mathcal{X}} \times \mathcal{Y}$ :

$$
\forall(\bar{x} \in \overline{\mathcal{X}}, y \in \mathcal{Y}): \bar{\psi}(\bar{x}, y)=\inf _{f, t, \bar{u}}\left\{f^{T} y+t: \bar{P} f+t \bar{p}+\bar{Q} \bar{u}+\bar{R} \bar{x} \leq_{\overline{\mathbf{K}}} \bar{s}\right\} .
$$

Let also

$$
x \mapsto X(x): \mathcal{X} \mapsto \overline{\mathcal{X}}
$$

be a $\mathbf{U}$-convex mapping such that the intersection of the $\mathbf{U}$-epigraph of the mapping with $\mathcal{X} \times \overline{\mathcal{X}}$ admits $\mathcal{K}$-representation:

$$
\left\{(x, \bar{x}): x \in \mathcal{X}, \bar{x} \in \overline{\mathcal{X}}, \bar{x} \geq_{\mathbf{U}} X(x)\right\}=\left\{(x, \bar{x}): \exists v: A x+B \bar{x}+C v \leq_{\widehat{\mathbf{K}}} d\right\} .
$$

Then the function $\psi(x, y)=\bar{\psi}(X(x), y)$ admits $\mathcal{K}$-representation on $\mathcal{X} \times \mathcal{Y}$ :

$$
\begin{aligned}
& \forall(x \in \mathcal{X}, y \in \mathcal{Y}): \\
& \psi(x, y)=\bar{\psi}(X(x), y)=\inf _{\bar{x}}\left\{\bar{\psi}(\bar{x}, y): \bar{x} \in \overline{\mathcal{X}} \& \bar{x} \geq_{\mathbf{U}} X(x)\right\} \\
& =\inf _{f, t, \bar{u}, \bar{x}}\left\{f^{T} y+t: \begin{array}{l}
\bar{x} \in \overline{\mathcal{X}}, \bar{x} \geq \mathbf{U} \\
\bar{P} f+t \bar{p}+\bar{Q} \bar{u}+\bar{R} \bar{x} \leq_{\overline{\mathbf{K}}} \bar{s}
\end{array}\right\} \\
& =\inf _{f, t, \bar{u}, \bar{x}, v}\left\{f^{T} y+t: \begin{array}{l}
A x+B \bar{x}+C v \leq_{\widehat{\mathbf{K}}} d \\
\bar{P} f+t \bar{p}+\bar{Q} \bar{u}+\bar{R} \bar{x} \leq_{\overline{\mathbf{K}}} \bar{s}
\end{array}\right\} \\
& =\inf _{f, t, u=[\bar{u}, \bar{x}, v]}\{f^{T} y+t: \underbrace{\left[\begin{array}{l}
A x+B \bar{x}+C v \leq \widehat{\mathbf{K}} d \\
\bar{P} f+t \bar{p}+\bar{Q} \bar{u}+\bar{R} \bar{x} \leq \overline{\mathbf{K}} \bar{s}
\end{array}\right]}_{\Leftrightarrow P f+t p+Q u+R x \leq \mathbf{K} s \text { with } \mathbf{K} \in \mathcal{K}}\} .
\end{aligned}
$$


6. [Partial maximization] Let

$$
\begin{aligned}
& \forall(x \in \mathcal{X}, y=[w ; z] \in \mathcal{Y}): \\
& \psi(x,[w ; z])=\inf _{[g ; h], \tau, u}\left\{g^{T} w+h^{T} z+\tau: G g+H h+\tau p+Q u+R x \leq_{\mathbf{K}} s\right\} \quad[\mathbf{K} \in \mathcal{K}],
\end{aligned}
$$

and let $\mathcal{Y}$ be compact and given by $\mathcal{K}$-representation:

$$
\mathcal{Y}=\left\{[w ; z]: \exists v: A w+B z+C v \leq_{\mathbf{L}} r\right\}
$$

such that the conic constraint $B z+C v \leq_{\mathbf{L}} r-A w$ in variables $z, v$ is essentially strictly feasible for every $w \in \mathcal{W}=\{w: \exists z:[w ; z] \in \mathcal{Y}\}$. Then the function

$$
\bar{\psi}(x ; w):=\max _{z}\{\psi(x,[w ; z]):[w ; z] \in \mathcal{Y}\}: \mathcal{X} \times \mathcal{W} \rightarrow \mathbf{R}
$$

is $\mathcal{K}$-representable provided it is continuous 5

$$
\begin{aligned}
& \forall(x \in \mathcal{X}, w \in \mathcal{W}): \\
& \bar{\psi}(x ; w)=\max _{z}\left\{\inf _{[g ; h], \tau, u}\left[g^{T} w+h^{T} z+\tau: G g+H h+\tau p+Q u+R x \leq_{\mathbf{K}} s\right]:[w ; z] \in \mathcal{Y}\right\} \\
& =\inf _{[g ; h], \tau, u}\left\{\max _{z}\left[g^{T} w+h^{T} z+\tau:[w ; z] \in \mathcal{Y}\right]: G g+H h+\tau p+Q u+R x \leq_{\mathbf{K}} s\right\} \\
& \quad \text { by the Sion-Kakutani Theorem; note that for } w \in \mathcal{W} \\
& \quad \text { the set }\{z:[w ; z] \in \mathcal{Y}\} \text { is nonempty, convex and compact] } \\
& =\inf _{[g ; h], \tau, u}\left\{\max _{z, v}\left[g^{T} w+h^{T} z+\tau: B z+C v \leq_{\mathbf{L}} r-A w\right]: G g+H h+\tau p+Q u+R x \leq_{\mathbf{K}} s\right\} \\
& =\inf _{[g ; h], \tau, u}\left\{\min _{\xi}\left[g^{T} w+(r-A w)^{T} \xi+\tau: B^{T} \xi=h, C^{T} \xi=0, \xi \geq_{\mathbf{L}^{*}} 0\right]:\right. \\
& \left.G g+H h+\tau p+Q u+R x \leq_{\mathbf{K}} s\right\} \\
& =\inf _{f, t, \bar{u}=[g, h, \tau, u, \xi]}\{\underbrace{[\text { by conic duality }]}_{\Leftrightarrow \bar{P} f+t \bar{p}+\bar{Q} \bar{u}+\bar{R} x \leq \overline{\mathbf{K}}^{\bar{s}} \text { with } \overline{\mathbf{K}} \in \mathcal{K}}
\end{aligned}
$$

Note that the last three rules combine with symmetry to induce "symmetric" rules on perspective transformation and superposition in $y$-variable and partial minimization in $x$-variable.

7. [Taking Fenchel conjugate] Let $\mathcal{X} \subset \mathbf{R}^{n}, \mathcal{Y} \subset \mathbf{R}^{m}$ be nonempty convex compact sets given by essentially strictly feasible $\mathcal{K}$-representations

$$
\mathcal{X}=\left\{x: \exists \xi: A x+B \xi \leq_{\mathbf{K}_{\mathcal{X}}} c\right\}, \mathcal{Y}=\left\{y: \exists \eta: C y+D \eta \leq_{\mathbf{K}_{\mathcal{Y}}} e\right\},
$$

and assume that the conic constraint

$$
D^{T} \lambda=0 \& \lambda \geq_{\mathbf{K}_{\mathcal{Y}}^{*}} 0
$$

is essentially strictly feasible (this definitely is the case when $\mathbf{K}_{\mathcal{Y}}$ is polyhedral). Let, next, $\psi(x, y): \mathcal{X} \times$ $\mathcal{Y} \rightarrow \mathbf{R}$ be continuous convex-concave function given by essentially strictly feasible $\mathcal{K}$-representation:

$$
\psi(x, y)=\inf _{f, t, u}\left\{f^{T} y+t: P f+t \Pi+Q u+R x \leq_{\mathbf{K}} s\right\} .
$$

Consider the Fenchel conjugate of $\psi$ - the function

$$
\psi_{*}(p, q)=\max _{x \in \mathcal{X}} \min _{y \in \mathcal{Y}}\left[p^{T} x+q^{T} y-\psi(x, y)\right]: \mathbf{R}^{n} \times \mathbf{R}^{m} \rightarrow \mathbf{R} .
$$

\footnotetext{
${ }^{5}$ Representation to follow holds true without the latter assumption; we make it to stay consistent with our general definition of representability, where the convex-concave function in question is assumed to be continuous.
} 
We claim that $\psi_{*}$ is a continuous convex-concave $\mathcal{K}$-representable function with $\mathcal{K}$-representation readily given by the $\mathcal{K}$-representations of $\mathcal{X}, \mathcal{Y}, \psi$

The fact that $\psi_{*}$ is well defined and continuous is readily given by compactness of $\mathcal{X}, \mathcal{Y}$ and continuity of $\psi$. These properties of the data imply by the Sion-Kakutani Theorem that

$$
\psi_{*}(p, q)=\min _{y \in \mathcal{Y}} \max _{x \in \mathcal{X}}\left[p^{T} x+q^{T} y-\psi(x, y)\right] .
$$

From the initial $\max _{x} \min _{y}$ definition of $\psi_{*}$ it follows that

$$
\psi_{*}(p, q)=\max _{x \in \mathcal{X}}\left[p^{T} x+\min _{y \in \mathcal{Y}}\left[q^{T} y-\psi(x, y)\right]\right]
$$

is the pointwise maximum of a family of affine functions of $p$ and thus is convex in $p$. From the $\min _{y} \max _{x}$ representation of $\psi_{*}$ it follows that

$$
\psi_{*}(p, q)=\min _{y \in \mathcal{Y}}\left[q^{T} y+\max _{x \in \mathcal{X}}\left[p^{T} x-\psi(x, y)\right]\right]
$$

is the pointwise minimum of a family of affine functions of $q$ and thus is concave in $q$.

It remains to build $\mathcal{K}$-representation of $\psi_{*}$. We have

$$
\begin{aligned}
\psi_{*}(p, q) & =\max _{x \in \mathcal{X}} \min _{y \in \mathcal{Y}}\left[p^{T} x+q^{T} y-\psi(x, y)\right] \\
= & \max _{x \in \mathcal{X}}\left[p^{T} x+\min _{y \in \mathcal{Y}} \sup _{f, t, u}\left\{[q-f]^{T} y-t: P f+t \Pi+Q u+R x \leq_{\mathbf{K}} s\right\}\right] \\
= & \max _{x \in \mathcal{X}}\left[p^{T} x+\sup _{f, t, u}\left\{\min _{y \in \mathcal{Y}}\left\{[q-f]^{T} y\right\}-t: P f+t \Pi+Q u+R x \leq_{\mathbf{K}} s\right\}\right]
\end{aligned}
$$

[by the Sion-Kakutani Theorem; recall that $\mathcal{Y}$ is convex and compact

and $[q-f]^{T} y-t$ is concave in $f, t, u$ and convex in $\left.y\right]$

$$
\begin{array}{r}
=\max _{x \in \mathcal{X}}\left[p^{T} x+\sup _{f, t, u}\left\{\sup _{\lambda}\left\{-e^{T} \lambda: f-C^{T} \lambda=q, D^{T} \lambda=0, \lambda \geq \mathbf{K}_{\mathcal{Y}}^{*} 0\right\}-t:\right.\right. \\
\left.\left.P f+t \Pi+Q u+R x \leq_{\mathbf{K}} s\right\}\right]
\end{array}
$$

[by Conic Duality; note that $\mathcal{Y}$ is given by essentially strictly feasible $\mathcal{K}$-representation]

$$
\begin{aligned}
& =\sup _{x \in \mathcal{X}, f, t, u, \lambda}\left\{p^{T} x-t-e^{T} \lambda: \begin{array}{ll}
D^{T} \lambda=0, f-C^{T} \lambda=q, \lambda \geq \mathbf{K}_{\mathcal{Y}}^{*} 0 \\
P f+t \Pi+Q u+R x \leq_{\mathbf{K}} s
\end{array}\right. \\
& =\sup _{x, \xi, f, t, u, \lambda}\left\{\begin{array}{ll}
D^{T} \lambda=0 & (a) \\
f-C^{T} \lambda=q & (b) \\
p^{T} x-t-e^{T} \lambda: \begin{array}{ll}
(c) \\
\lambda \mathbf{K}_{\mathcal{Y}}^{*} 0
\end{array} \\
P f+t \Pi+Q u+R x \leq_{\mathbf{K}} s & (d) \\
A x+B \xi \leq_{\mathbf{K}_{\mathcal{X}}} c & (e)
\end{array}\right\}
\end{aligned}
$$

Now, the $\mathcal{K}$-representation of $\mathcal{X}$ is essentially strictly feasible, so that (e) admits an essentially strictly feasible solution $\bar{x}, \bar{\xi}$; observe that $\bar{x} \in \mathcal{X}$. The $\mathcal{K}$-representation of $\psi$ is essentially strictly feasible, implying that $\bar{x}$ can be augmented by $\bar{f}, \bar{t}, \bar{u}$ in such a way that $(\bar{x}, \bar{f}, \bar{t}, \bar{u})$ is an essentially strictly feasible solution to $(d)$. By the origin of constraints $(a)-(c)$, their system is feasible for all $f, q$ as a system of constraints on $\lambda$, Besides this, by assumption, there exists a representation $\mathbf{K}_{\mathcal{Y}}^{*}=M \times N$ with regular cone $M$ and polyhedral cone $N$ and $\lambda^{\prime} \in[$ int $M] \times N$ such that $D^{T} \lambda^{\prime}=0$. Taking into account that the system $(a)-(c)$, considered as a system in variable $\lambda$, is solvable for all $f, q$, there exists $\lambda^{\prime \prime} \in \mathbf{K}_{\mathcal{Y}^{*}}$ such that $D^{T} \lambda^{\prime \prime}=0$ and $\bar{f}-C^{T} \lambda^{\prime \prime}=q+C^{T} \lambda^{\prime}$. This implies that $\bar{\lambda}=\lambda^{\prime}+\lambda^{\prime \prime}$ taken together with $\bar{f}$ is an essentially strictly feasible solution to the system $(a)-(c)$ of constraints 
in variables $\lambda$ and $f$. The bottom line is that the constraints $(a)-(e)$ in variables $x, \xi, f, t, u, \lambda$ form an essentially strictly feasible conic constraint. Besides this, problem (8) is bounded by its origin. By Conic Duality we conclude from (8) that

$$
\begin{aligned}
\psi_{*}(p, q)=\min _{\alpha, \beta, \gamma, \delta, \epsilon}\left\{\begin{array}{r}
\gamma \geq \mathbf{K}_{\mathcal{Y}} 0, \delta \geq \mathbf{K}^{*} 0, \epsilon \mathbf{K}_{\mathcal{X}}^{*} 0 \\
\beta^{T} q+s^{T} \delta+c^{T} \epsilon: \begin{array}{l}
R^{T} \delta+A^{T} \epsilon=p, B^{T} \epsilon=0, P^{T} \delta+\beta=0 \\
\Pi^{T} \delta=-1, Q^{T} \delta=0, D \alpha-C \beta+e=\gamma
\end{array}
\end{array}\right. \\
=\min _{\beta, \tau, w=(\alpha, \delta, \epsilon)}\left\{\begin{array}{r}
\delta \geq \mathbf{K}^{*} 0, \epsilon \geq \mathbf{K}_{\mathcal{X}}^{*} 0, \tau=s^{T} \delta+c^{T} \epsilon \\
\beta^{T} q+\tau: \begin{array}{l}
R^{T} \delta+A^{T} \epsilon=p, B^{T} \epsilon=0, P^{T} \delta+\beta=0 \\
\Pi^{T} \delta=-1, Q^{T} \delta=0, D \alpha-C \beta+e \geq \mathbf{K}_{\mathcal{Y}} 0
\end{array}
\end{array}\right\}
\end{aligned}
$$

which is the desired $\mathcal{K}$-representation of $\psi_{*}$.

\subsection{Illustrations}

A. Our first illustration is motivated by a statistical application of saddle point optimization-near-optimal recovery of linear forms in Discrete observation scheme, see [10, Section 3.1]. Let

$$
\psi(x, y)=\ln \left(\sum_{i} \mathrm{e}^{x_{i}} y_{i}\right): \mathcal{X} \times \mathcal{Y} \rightarrow \mathbf{R}
$$

$\mathcal{X}$ and $\mathcal{Y}$ be $\mathcal{K}$-representable, and let $\mathcal{Y}, 0 \notin \mathcal{Y}$, be is a compact subset of the nonnegative orthant. Because for $z>0$

$$
\ln z=\inf _{u} z e^{u}-u-1
$$

for $y \geq 0$ we clearly have

$$
\begin{aligned}
\ln \left(\sum_{i} \mathrm{e}^{x_{i}} y_{i}\right)= & \inf _{u}\left[\left(\sum_{i} \mathrm{e}^{x_{i}} y_{i}\right) \mathrm{e}^{u}-u-1\right]=\inf _{f, u}\left[\sum_{i} y_{i} f_{i}-u-1: f_{i} \geq \mathrm{e}^{x_{i}+u}\right] \\
& =\inf _{f, t, u}\left\{f^{T} u+t: f_{i} \geq \mathrm{e}^{x_{i}+u} \forall i \& t \geq-u-1\right\}
\end{aligned}
$$

The resulting representation is a $\mathcal{K}$-representation, provided that the closed w.r.t. taking finite direct products and passing to the dual cone family $\mathcal{K}$ of regular cones contains $\mathbf{R}_{+}$, the exponential cone

$$
\mathbf{E}=\operatorname{cl}\left\{[t ; s ; r]: t \geq s \mathrm{e}^{r / s}, s>0\right\},
$$

and, therefore, its dual cone

$$
\mathbf{E}^{*}=\operatorname{cl}\{[\tau ; \sigma ;-\rho]: \tau>0, \rho>0, \sigma \geq \rho \ln (\rho / \tau)-\rho\}
$$

B. Let now

$$
\psi(x, y)=\left(\sum_{i=1}^{n} \theta_{i}^{p}(x) y_{i}\right)^{1 / p}
$$

where $p>1, \theta_{i}(x)$ are nonnegative $\mathcal{K}$-representable real-valued functions on $\mathcal{K}$-representable set $\mathcal{X}$, and $\mathcal{Y}$ is a $\mathcal{K}$-representable subset of the nonnegative orthant. In this case, as is easily seen, for all $(x \in \mathcal{X}, y \in \mathcal{Y})$ it holds

$$
\psi(x, y)=\inf _{[f ; t]}\left\{f^{T} y+t: t \geq 0, f \geq 0, t^{\frac{p-1}{p}} f_{i}^{\frac{1}{p}} \geq \kappa \theta_{i}(x), i \leq n\right\} \quad\left[\kappa=p^{-1}(p-1)^{\frac{p-1}{p}}\right]
$$

which can immediately be converted into $\mathcal{K}$-representation, provided $\mathcal{K}$ contains $3 \mathrm{D}$ Lorentz cone $\mathbf{L}^{3}=\{x \in$ $\left.\mathbf{R}^{3}: x_{3} \geq \sqrt{x_{1}^{2}+x_{2}^{2}}\right\}$ and $p$ is rational, see [4, Section 3.3] or [5, Section 2.3.5].

C. In our next example, $\mathcal{X} \subset \mathbf{R}^{m \times n}$ and $\mathcal{Y} \subset \mathbf{S}_{+}^{m}$ are nonempty convex sets, and

$$
\psi(x, y)=2 \sqrt{\operatorname{Tr}\left(x^{T} y x\right)}: \mathcal{X} \times \mathcal{Y} \rightarrow \mathbf{R} .
$$


Taking into account that for $a \geq 0$ one has $2 \sqrt{a}=\inf _{s>0}[a / s+s]$, we have

$$
\begin{aligned}
& \forall(x \in \mathcal{X}, y \in \mathcal{Y}): \\
& \begin{aligned}
\psi(x, y)= & 2 \sqrt{\operatorname{Tr}\left(y\left[x x^{T}\right]\right)}=\inf _{g}\left\{2 \sqrt{\operatorname{Tr}(y g)}: g \succeq x x^{T}\right\} \\
& =\inf _{f, s}\left\{\operatorname{Tr}(y f)+s: s>0, f s \succeq x x^{T}\right\} \\
& =\inf _{f, s}\left\{\operatorname{Tr}(y f)+s:\left[\begin{array}{c|c}
f & x \\
\hline x^{T} & s I_{n}
\end{array}\right] \succeq 0\right\} .
\end{aligned}
\end{aligned}
$$

The resulting representation is $\mathcal{K}$-representation, provided that $\mathcal{K}$ contains semidefinite cones.

This is how $\mathbf{C}$ works in Robust Markowitz Portfolio Selection (cf, e.g., 6, 8]

$$
\min _{x \in \mathcal{X}} \max _{y \in \mathcal{Y}}\left[-r^{T} x+2 \rho \sqrt{x^{T} y x}\right]
$$

(here $x \in \mathbf{R}^{n}$ is the composition of portfolio, $r$ is the vector of expected returns, and $y$ is the uncertain covariance matrix of the returns). Assuming for the sake of definiteness that $\mathcal{Y}$ is cut off $\mathbf{S}_{+}^{n}$ by the constraints

$$
a_{i}^{T} y a_{i}+b_{i} y+y b_{i}^{T} \preceq p_{i}, i \leq I, y_{-} \leq y \leq y_{+}
$$

(where $\leq$ for matrices acts entrywise) and applying our machinery on the top of the above semidefinite representation of $2 \sqrt{x^{T} y x}$, the saddle point problem reduces to

$$
\begin{aligned}
\min _{x, s, \alpha_{i}, \mu_{ \pm}}\left\{\begin{array}{l|l}
-r^{T} x+\rho\left[s+\sum_{i} \operatorname{Tr}\left(\alpha_{i} p_{i}\right)+\operatorname{Tr}\left(\mu_{+} y_{+}-\mu_{-} y_{-}\right)\right]: \\
{\left[\begin{array}{l|l}
\sum_{i}\left[a_{i} \alpha_{i} a_{i}^{T}+\alpha_{i} b_{i}+b_{i}^{T} \alpha_{i}\right]+\mu_{+}-\mu_{-} & x \\
x^{T} & s
\end{array}\right] \succeq 0} \\
\alpha_{i} \succeq 0, i \leq I, \mu_{ \pm} \geq 0, x \in \mathcal{X}
\end{array}\right\} .
\end{aligned}
$$

D. In our concluding example, $\mathcal{K}$ contains the products of semidefinite cones, $\mathcal{X}=\mathbf{R}^{m \times n}, \mathcal{Y}=\mathbf{S}_{+}^{n}$, and

$$
\psi(x, y)=\operatorname{Tr}\left(x^{T} x y^{1 / 2}\right): \mathcal{X} \times \mathcal{Y} \rightarrow \mathbf{R} .
$$

This is a "generalized bilinear function"; in terms of item 4c of Section 3.4.1 we have $F(x)=x^{T} x$, $G(y)=y^{1 / 2}, \mathbf{U}=\mathbf{U}^{*}=\mathbf{S}_{+}^{n}$, and

$$
\begin{aligned}
\mathrm{Epi}_{\mathbf{U}} F & :=\left\{(x, z): z \succeq x^{T} x\right\}=\left\{(x, z):\left[\begin{array}{c|c}
z & x^{T} \\
\hline x & I_{m}
\end{array}\right] \succeq 0\right\}, \\
\mathrm{Hypo}_{\mathbf{U}^{*}} G & :=\left\{(y, w): y \in \mathbf{S}_{+}^{n}, w \preceq y^{1 / 2}\right\}=\left\{(y, w): \exists v:\left[\begin{array}{c|c}
y & v \\
\hline v & I_{n}
\end{array}\right] \succeq 0, v \succeq 0, w \preceq v\right\} .
\end{aligned}
$$

With these data, the construction from item 4. c of Section 3.4.1 leads straightforwardly to the following semidefinite representation of $\psi$ :

$$
\forall\left(x \in \mathbf{R}^{m \times n}, y \in \mathbf{S}_{+}^{n}\right): \psi(x, y):=\operatorname{Tr}\left(x^{T} x y^{1 / 2}\right)=\inf _{f, t, u=(z, \beta, \gamma)}\left\{\begin{array}{r}
f \in \mathbf{S}^{n}, \beta \in \mathbf{R}^{n \times n}, \gamma \in \mathbf{S}^{n}, z \in \mathbf{S}^{n} \\
\left.\operatorname{Tr}(f y)+t: \begin{array}{c|c|c|c|c|c|}
t=\operatorname{Tr}(\gamma), z \preceq \beta+\beta^{T} \\
{\left[\beta^{T}\right.} & \gamma
\end{array}\right] \succeq 0,\left[\begin{array}{l|l}
z & x^{T} \\
\hline x & I_{m}
\end{array}\right] \succeq 0
\end{array}\right\} .
$$

\section{Well-structured variational inequalities with monotone opera- tors}

\subsection{Preliminaries}

Let $\mathcal{X}$ be a nonempty convex subset of Euclidean space $E$. A vector field $F(x): x \rightarrow E$ is called monotone, if

$$
\langle F(x)-F(y), x-y\rangle \geq 0 \forall x, y \in \mathcal{X}
$$


Variational Inequality $\mathrm{VI}(F, \mathcal{X})$ reads

$$
\text { Find } x_{*} \in \mathcal{X}:\left\langle F(y), x_{*}-y\right\rangle \leq 0 \forall y \in \mathcal{X} .
$$

Solutions which are sought in $\operatorname{VI}(F, \mathcal{X})$ are called weak solutions; they do exist whenever $\mathcal{X}$ is a compact set and $F$ is monotone on $\mathcal{X}$. Strong solutions are points $x_{*} \in \mathcal{X}$ such that

$$
\left\langle F\left(x_{*}\right), x-x_{*}\right\rangle \geq 0 \forall x \in \mathcal{X} ;
$$

from monotonicity of $F$ it follows that every strong solution is a weak one. The opposite is true provided that $F$ is continuous on $\mathcal{X}$.

A natural (in)accuracy measure for a candidate approximate solution to $\operatorname{VI}(F, \mathcal{X})$ is the dual gap function

$$
\epsilon_{\mathrm{VI}}[x \mid \mathcal{X}]=\sup _{y \in \mathcal{X}}\langle F(y), x-y\rangle
$$

this function is nonnegative and is zero exactly at weak solutions to $V I(F, \mathcal{X})$.

Our current goal is to develop a framework for converting "well-structured" VI's with monotone vector fields into the usual conic problems, thus bringing them within the grasp of Interior-Point polynomial time methods and software like CVX. What follows can be seen as a streamlined "well-structured" (i.e., conic representation-oriented) extension of what in [13. Section 7.4] was called a convex representation of a monotone operator.

\subsection{Conic representability of monotone vector fields and monotone VI's in conic form}

\subsubsection{Conic representation of a monotone vector field}

Let $F(x): \mathcal{X} \rightarrow E$ be a monotone and continuous vector field on nonempty convex subset $\mathcal{X}$ of Euclidean space $E$. Consider the set

$$
\mathcal{F}[F, \mathcal{X}]=\{[t ; g ; x] \in \mathbf{R} \times E \times E: x \in \mathcal{X}, t-\langle g, y\rangle \geq\langle F(y), x-y\rangle \forall y \in \mathcal{X}\}
$$

and let us make several straightforward observations.

4.2.1. A. $\mathcal{F}[F, \mathcal{X}]$ is a convex set which contains all triples $[\langle F(x), x\rangle ; F(x) ; x], x \in \mathcal{X}$; this set is closed provided $\mathcal{X}$ is so. Besides this, $\mathcal{F}[F, \mathcal{X}]$ is $t$-monotone:

$$
[t ; g ; x] \in \mathcal{F}[F, \mathcal{X}] \text { and } t^{\prime} \geq t \Rightarrow\left[t^{\prime} ; g ; x\right] \in \mathcal{F}[F, \mathcal{X}] .
$$

Indeed, $\mathcal{F}[F, \mathcal{X}]$ is the intersection of solution set of the system of nonstrict linear constraints

$$
t-\langle g, y\rangle-\langle F(y), x\rangle \geq-\langle F(y), y\rangle, y \in \mathcal{X}
$$

in variables $g, t, x$ (this set is closed and convex) with the convex set $\{[t ; g ; x]: x \in \mathcal{X}\}$ (which is closed if so is $\mathcal{X})$. By monotonicity, for every $x \in \mathcal{X}$ we have

$$
\langle F(x), x-y\rangle \geq\langle F(y), x-y\rangle \forall y \in \mathcal{X},
$$

so that $[\langle F(x), x\rangle ; F(x) ; x] \in \mathcal{F}[F, \mathcal{X}] ;$ and $t$-monotonicity is evident.

4.2.1.B. For $\epsilon \geq 0$, let

$$
\mathcal{X}_{*}(\epsilon)=\left\{[g ; t] \in E \times \mathbf{R}: \sup _{y \in \mathcal{X}}[t-\langle g, y\rangle] \leq \epsilon\right\},
$$

so that $\mathcal{X}_{*}(\epsilon)$ is a nonempty closed convex set. Then for every $\epsilon \geq 0, \epsilon$-solutions to $\operatorname{VI}(F, \mathcal{X})$ - points $x \in \mathcal{X}$ such that

$$
\epsilon_{\mathrm{VI}}[x \mid F]:=\sup _{y \in \mathcal{X}}\langle F(y), x-y\rangle \leq \epsilon
$$

are exactly the points

$$
x: \exists(t, g):[t ; g ; x] \in \mathcal{F}[F, \mathcal{X}] \text { and }[t ; g] \in \mathcal{X}_{*}(\epsilon) \text {; }
$$


Indeed, let $x, t, g$ be such that $[t ; g ; x] \in \mathcal{F}[F, \mathcal{X}]$ and $[t ; g] \in \mathcal{X}_{*}(\epsilon)$. From the first inclusion it follows that $x \in \mathcal{X}$ and

$$
t-\langle g, y\rangle \geq\langle F(y), x-y\rangle \forall y \in \mathcal{X}
$$

while from the second inclusion it follows that $t-\langle g, y\rangle \leq \epsilon$ for all $y \in \mathcal{X}$. Taken together, these two relations imply that $x \in \mathcal{X}$ and $\langle F(y), x-y\rangle \leq \epsilon$ for all $y \in \mathcal{X}$, i.e., $\epsilon_{\mathrm{VI}}(x \mid F) \leq \epsilon$. Vice versa, if $x \in \mathcal{X}, \epsilon \geq 0$, and $\epsilon_{\mathrm{VI}}[x \mid F] \leq \epsilon$, then the triple $t=\epsilon, g=0, x$ clearly belongs to $\mathcal{F}[F, \mathcal{X}]$ and $[t ; g] \in \mathcal{X}_{*}(\epsilon)$.

$\mathcal{K}$-representation of $(F, \mathcal{X})$. Given a continuous monotone vector field $F: \mathcal{X} \rightarrow E$ on a nonempty convex subset $\mathcal{X}$ of Euclidean space $E$, let us call a conic constraint 6

$$
X x+G g+t T+U u \leq_{\mathbf{K}} a
$$

in variables $t \in \mathbf{R}, g \in E, x \in E$, and $u \in \mathbf{R}^{k}$ a $\mathcal{K}$-representation of $(F, \mathcal{X})$, if the set

$$
\mathcal{T}:=\left\{[t ; g ; x]: \exists u: X x+G g+t T+U u \leq_{\mathbf{K}} a\right\}
$$

possesses the following two properties:

(i) $\mathcal{T}$ is contained in $\mathcal{F}[F, \mathcal{X}]$ and, along with the latter set, is "t-monotone:"

$$
[t ; g ; x] \in \mathcal{T} \text { and } t^{\prime} \geq t \Rightarrow\left[t^{\prime} ; g ; x\right] \in \mathcal{T}
$$

(note that when $\mathcal{T} \neq \emptyset, t$-monotonicity is equivalent to $T \leq_{\mathbf{K}} 0$ );

and

(ii) $\mathcal{T}$ contains the set

$$
\{[\langle F(x), x\rangle ; F(x) ; x], x \in \mathcal{X}\}
$$

If the set (13) satisfies (i) and the relaxed version of (ii), specifically,

(ii') $\mathcal{T}$ contains the set

$$
\{[t ; F(x) ; x]: t>\langle F(x), x\rangle, x \in \mathcal{X}\} \quad[\subset \mathcal{F}[F, \mathcal{X}]]
$$

we say that (12) is an almost $\mathcal{K}$-representation of $(F, \mathcal{X})$.

Let us make an immediate observation:

Remark 4.1 In the situation described in the beginning of this section, let $\mathcal{X}$ be closed, and let $\mathcal{Y}$ be a convex set such that

$$
\operatorname{Conv}\left\{\left[x^{T} F(x) ; F(x) ; x\right], x \in \mathcal{X}\right\} \subset \mathcal{Y} \subset \operatorname{cl} \operatorname{Conv}\left\{\left[x^{T} F(x) ; F(x) ; x\right], x \in \mathcal{X}\right\}
$$

Then every $\mathcal{K}$-representation of $\mathcal{Y}$ represents $(F, \mathcal{X})$.

\subsubsection{Conic form of conic-representable monotone VI}

Our main observation is as follows:

\footnotetext{
${ }^{6}$ Recall that we have fixed a family $\mathcal{K}$ of regular cones in Euclidean spaces, and by our standing convention, all cones to be considered below belong to $\mathcal{K}$.
} 
Proposition 4.1 Let $\mathcal{X} \subset E$ be nonempty convex compact given by essentially strictly feasible $\mathcal{K}$ representation:

$$
\mathcal{X}=\left\{x: \exists v: A x+B v \leq_{\mathbf{L}} b\right\}, \quad[\mathbf{L} \in \mathcal{K}],
$$

so that $\mathcal{X}_{*}(\epsilon)$, see (10), by conic quality, admits $\mathcal{K}$-representation as follows:

$$
\mathcal{X}_{*}(\epsilon)=\left\{[t ; g]: \exists \lambda: A^{T} \lambda+g=0, B^{T} \lambda=0, t+b^{T} \lambda \leq \epsilon, \lambda \geq \mathbf{L}^{*} 0\right\} .
$$

Let, moreover, $F: \mathcal{X} \rightarrow E$ be a continuous monotone vector field, and let (12) be an almost $\mathcal{K}$-representation of $(F, \mathcal{X})$. Then for every $\epsilon>0$ the system of conic constraints

$$
\begin{array}{rcll}
X x+G g+t T+U u & \leq_{\mathbf{K}} & a & (a) \\
A^{T} \lambda+g & = & 0 & (b .1) \\
B^{T} \lambda & = & 0 & (b .2) \\
t+b^{T} \lambda & \leq & \epsilon & (b .3) \\
\lambda & \geq \mathbf{L}^{*} & 0 & (b .4)
\end{array}
$$

in variables $x, g, t, u$, and $\lambda$ is feasible, and $x$-component of any feasible solution belongs to $\mathcal{X}$ and is an $\epsilon$-solution to $\mathrm{VI}(F, \mathcal{X})$ :

$$
\epsilon_{\mathrm{VI}}[x \mid F] \leq \epsilon .
$$

Therefore, finding an $\epsilon$-solution to $\operatorname{VI}(F, \mathcal{X})$ reduces to finding a feasible solution to an explicit $\mathcal{K}$-conic constraint.

When (12) is a $\mathcal{K}$-representation of $(F, \mathcal{X})$, the above conclusion holds true for all $\epsilon \geq 0$.

Proof. Let us fix $\epsilon>0$, and let $\bar{x}$ solve $\operatorname{VI}(F, X)$ (a solution exists since $\mathcal{X}$ is compact). Then $\langle F(\bar{x}), \bar{x}-y\rangle \leq 0$ for all $y \in \mathcal{X}$ due to continuity of $F$ on $\mathcal{X}$. Consequently, given $\epsilon>0$, setting $\bar{t}=\langle F(\bar{x}), \bar{x}\rangle, \bar{g}=F(\bar{x})$, we have $[\bar{t}+\epsilon ; \bar{g} ; \bar{x}] \in \mathcal{X}_{*}(\epsilon)$, implying by (15) that there exists $\bar{\lambda}$ such that $t=\bar{t}+\epsilon, g=\bar{g}, \lambda=\bar{\lambda}$ solve (16. $b .1-4)$. Besides this, by (ii') there exists $\bar{u}$ such that $x=\bar{x}, g=\bar{g}, t=\bar{t}+\epsilon, u=\bar{u}$ solve (16) $a$ ). Thus, (16) is feasible.

Next, if $x, g, t, u, \lambda$ is a feasible solution to (16), then by (16, $a$ ) one has $[t ; g ; x] \in \mathcal{T}$, with $\mathcal{T}$ given by (13), whence $[t ; g ; x] \in \mathcal{F}[F, \mathcal{X}]$, implying that $x \in \mathcal{X}$, see (9). Besides this, (16, b.1-4) say that $[t ; g] \in \mathcal{X}_{*}(\epsilon)$. Thus, $[t ; g ; x] \in \mathcal{F}[F, \mathcal{X}]$ and $[t ; g] \in \mathcal{X}_{*}(\epsilon)$, implying by 4.2.1, B that $\epsilon_{\mathrm{VI}}[x \mid F] \leq \epsilon$.

Finally, when (12) is a $\mathcal{K}$-representation of $(F, \mathcal{X})$, the above reasoning works for $\epsilon=0$.

\subsection{Calculus of conic representations of monotone vector fields}

$\mathcal{K}$-representations of pairs $(F, \mathcal{X})(\mathcal{X}$ is a nonempty convex subset of Euclidean space $E, F: \mathcal{X} \rightarrow E$ is a continuous monotone vector field) admit a calculus; for verification of claims to follow, see Appendix $\mathrm{A}$

\subsubsection{Raw materials}

Raw materials for the calculus of $\mathcal{K}$-representable monotone vector fields include:

1. [Affine monotone vector field] Let $\mathcal{K}$ contain all Lorentz cones. Then affine monotone vector field $F(x)=A x+a$ on $\mathcal{X}=E=\mathbf{R}^{n}$ is $\mathcal{K}$-represented by conic constraint (cf. [13, Proposition 7.4.3])

$$
t \geq x^{T} \bar{A} x+a^{T} x, g=A x+a
$$

in variables $t, g, x$, where $\bar{A}=\frac{1}{2}\left[A+A^{T}\right]$.

2. [Gradient field of continuously differentiable $\mathcal{K}$-representable convex function] Let $\mathcal{X}$ be nonempty convex compact set, and $f(x): \mathcal{X} \rightarrow \mathbf{R}$ be continuously differentiable convex function with $\mathcal{K}$-representable epigraph,

$$
\{(x, s): s \geq f(x), x \in \mathcal{X}\}=\left\{(x, s): \exists u: A x+s p+Q u \leq_{\mathbf{K}} a\right\},
$$

the representation being essentially strictly feasible. Then the conic constraint

$$
t \geq s+r, A x+s p+Q u \leq_{\mathbf{K}} a, r \geq a^{T} \lambda, A^{T} \lambda=g, p^{T} \lambda=-1, Q^{T} \lambda=0, \lambda \geq \mathbf{K}^{*} 0
$$

in variables $t, g, x, s, r, \lambda$, and $u$ represents $\left(F(\cdot):=f^{\prime}(\cdot), \mathcal{X}\right)$ (cf. [13, Proposition 7.4.4]). 
3. [Monotone vector field associated with continuously differentiable $\mathcal{K}$-representable convex-concave function $\psi$ ] Let $\mathcal{K}$ contain the 3D Lorentz cone, and let $\mathcal{U} \subset \mathbf{R}^{n_{u}}$ and $\mathcal{V} \subset \mathbf{R}^{n_{v}}$ be $\mathcal{K}$-representable nonempty compact sets:

$$
\begin{aligned}
& \mathcal{U}=\left\{u: \exists \alpha: A u+B \alpha \leq_{\mathbf{K}} a\right\}, \\
& \mathcal{V}=\left\{v: \exists \beta: C v+D \beta \leq_{\mathbf{L}} b\right\},
\end{aligned}
$$

both representations being essentially strictly feasible. Assume that $\psi(u, v): \mathcal{U} \times \mathcal{V} \mapsto \mathbf{R}$ is a continuously differentiable convex-concave function which is $\mathcal{K}$-representable on $\mathcal{U} \times \mathcal{V}$ with essentially strictly feasible representation (see Section 3.1); that is, $\psi$ admits representation

$$
\forall(u \in \mathcal{U}, v \in \mathcal{V}): \psi(u, v)=\inf _{f, \tau, \xi}\left\{f^{T} v+\tau: P f+\tau p+Q \xi+R u \leq_{\mathbf{M}} c\right\}
$$

such that the conic constraint $P f+\tau p+Q \xi \leq_{\mathbf{M}} c-R u$ in variables $f, \tau, \xi$ is essentially strictly feasible for every $u \in \mathcal{U}$. Let

$$
F(u, v)=\left[\nabla_{u} \psi(u, v) ;-\nabla_{v} \psi(u, v)\right]: \mathcal{X}:=\mathcal{U} \times \mathcal{V} \rightarrow E:=\mathbf{R}^{n_{u}} \times \mathbf{R}^{n_{v}}
$$

be the vector field associated with $\mathcal{U}, \mathcal{V}, \psi$; it is well known that this field is monotone. Then

(i) The set

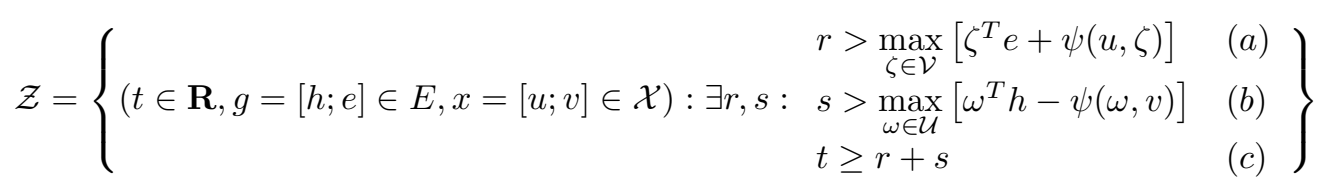

satisfies the relations

$$
\begin{array}{rlll}
\mathcal{Z} & \subset & \mathcal{F}[F, \mathcal{X}] & (a) \\
x \in \mathcal{X}, g=F(x), t>x^{T} F(x) & \Rightarrow & {[t ; g ; x] \in \mathcal{Z}}
\end{array}
$$

(ii) Besides this, $\mathcal{Z}$ is nothing but the projection on the plane of $(t, g=[h ; e], x=[u ; v])$-variables of the solution set of the conic constraint

$$
\left[\begin{array}{l}
t \geq r+s, r \geq \theta+\gamma^{T} b+\tau, s \geq \theta^{\prime}+c^{T} \delta+a^{T} \epsilon \\
A u+B \alpha \leq_{\mathbf{K}} a, C v+D \beta \leq_{\mathbf{L}} b, P f+\tau p+Q \xi+R u \leq_{\mathbf{M}} c \\
C^{T} \gamma=f+e, D^{T} \gamma=0, P^{T} \delta+v=0, p^{T} \delta=-1, Q^{T} \delta=0, R^{T} \delta+A^{T} \epsilon=h, B^{T} \epsilon=0, \\
\gamma \geq_{\mathbf{L}^{*}} 0, \delta \geq_{\mathbf{M}^{*}} 0, \epsilon \mathbf{K}^{*} 0, \eta \geq 0, \theta \geq 0, \eta \theta \geq 1, \eta^{\prime} \geq 0, \theta^{\prime} \geq 0, \eta^{\prime} \theta^{\prime} \geq 1
\end{array}\right]
$$

in variables $t, g=[h ; e], x=[u ; v], r, s, f, \tau, \xi, \alpha, \beta, \gamma, \eta, \theta, \delta, \epsilon, \eta^{\prime}$, and $\theta^{\prime} 7$

Taken together, $(i)$ and (ii) say that the conic constraint (24) almost represents $(F, \mathcal{X})$.

4. [Univariate monotone rational vector field] Let $\mathcal{X}=[a, b] \subset \mathbf{R}(-\infty<a<b<\infty)$, and let $\alpha(x)$ and $\beta(x)$ be real algebraic polynomials such that $\beta(x)>0$ on $\mathcal{X}$. Suppose that the univariate vector field given on $\mathcal{X}$ by the function $F(x)=\frac{\alpha(x)}{\beta(x)}$ is nondecreasing on $\mathcal{X}$, and that $\mathcal{K}$ contain all semidefinite cones. Then the set

$$
\mathcal{Y}:=\operatorname{Conv}\{[x F(x) ; F(x) ; x], x \in \mathcal{X}\}
$$

admits explicit $\mathcal{K}$-representation which, by Remark 4.1, represents $(F, \mathcal{X})$.

\footnotetext{
${ }^{7}$ When one of the authors was a freshman at Math. Department of Moscow State University, his mate left lecture on Linear Algebra writing in his notebook: "The lecture was terminated due to shortage of indices." The list of variables in (24) comes close to this natural limit...
} 


\subsubsection{Calculus rules}

Calculus rules are as follows.

1. [Restriction on a $\mathcal{K}$-representable set] Let conic constraint

$$
X x+G g+t T+U u \leq_{\mathbf{K}} a
$$

in variables $t, g, x, u$ represent (almost represent) $(F, \mathcal{X})$, and let set $\mathcal{Y} \subset E$ be $\mathcal{K}$-representable:

$$
\mathcal{Y}=\left\{y \in E: \exists v: A y+B v \leq_{\mathbf{L}} b\right\}
$$

and have a nonempty intersection $\mathcal{Z}=\mathcal{Y} \cap \mathcal{X}$ with $\mathcal{X}$. Denoting by $\bar{F}$ the restriction of $F$ on $\mathcal{Z}$, the conic constraint

$$
\left\{X x+G g+t T+U u \leq_{\mathbf{K}} a, A x+B v \leq_{\mathbf{L}} b\right\}
$$

in variables $t, g, x, u, v$ represents (resp., almost represent) $(\bar{F}, \mathcal{Z})$ (cf. [13, Proposition 7.4.5]).

2. [Direct summation] For $k \leq K$, let $\mathcal{X}_{k}$ be nonempty convex subsets of Euclidean spaces $E_{k}$ and $F_{k}: \mathcal{X}_{k} \rightarrow E_{k}$ be continuous monotone vector fields, and let

$$
X_{k} x+G_{k} g_{k}+t_{k} T_{k}+U_{k} u_{k} \leq_{\mathbf{K}_{k}} a_{k}, 1 \leq k \leq K
$$

be $\mathcal{K}$-representations of $\left(F_{k}, \mathcal{X}_{k}\right)$. Denote

$$
F\left(\left[x_{1} ; \ldots ; x_{K}\right]\right)=\left[F_{1}\left(x_{1}\right) ; \ldots ; F_{K}\left(x_{K}\right)\right] \text { and } \mathcal{X}=\mathcal{X}_{1} \times \ldots \times \mathcal{X}_{K} .
$$

Then the conic constraint

$$
\begin{array}{rlll}
X_{k} x_{k}+G_{k} g_{k}+t_{k} T_{k}+U_{k} u_{k} & \leq_{\mathbf{K}_{k}} & a_{k}, k \leq K \\
t & = & \sum_{k} t_{k} & (a)
\end{array}
$$

in variables $t, g:=\left[g_{1} ; \ldots ; g_{K}\right], x:=\left[x_{1} ; \ldots ; x_{K}\right], t_{1}, \ldots, t_{K}, u_{1}, \ldots, u_{K} \mathcal{K}$-represents $(F, \mathcal{X})$.

When conic constraints (28) almost represent $\left(F_{k}, \mathcal{X}_{k}\right)$, (29) almost represents $(F, \mathcal{X})$ (cf. [13, Proposition 7.4.6]).

3. [Taking conic combinations] Let $\mathcal{X}$ be a nonempty convex subset of Euclidean space $E$, and let $F_{1}, \ldots, F_{K}$ be continuous monotone vector fields on $X$ with $\left(F_{k}, \mathcal{X}\right)$ admitting $\mathcal{K}$-representations

$$
X_{k} x+G_{k} g+t T_{k}+U_{k} u_{k} \leq_{\mathbf{K}_{k}} a_{k}, 1 \leq k \leq K .
$$

Let, further, $\alpha_{k}>0$ be given, and let

$$
F(x)=\sum_{k} \alpha_{k} F_{k}(x): \mathcal{X} \rightarrow E
$$

The conic constraint

$$
\begin{aligned}
& X_{k} x+G_{k} g_{k}+t_{k} T_{k}+U_{k} u_{k} \leq \mathbf{K}_{k} a_{k}, 1 \leq k \leq K \\
& g=\sum_{k} \alpha_{k} g_{k} \\
& t=\sum_{k} \alpha_{k} t_{k}
\end{aligned}
$$

in variables $t, g, x,\left\{t_{k}, g_{k}, u_{k}, k \leq K\right\}$ is a $\mathcal{K}$-representation of $(F, \mathcal{X})$.

When (30) are almost representations of $\left(F_{k}, \mathcal{X}\right)$, (31) is almost representation of $(F, \mathcal{X})$.

4. [Affine substitution of variables] Let $\mathcal{X}$ be a nonempty convex subset of $E, F$ be a continuous monotone vector field on $X$, with $(F, \mathcal{X})$ given by $\mathcal{K}$-representation

$$
X x+G g+t T+U u \leq_{\mathbf{K}} a .
$$


Assume that $\xi \mapsto A \xi+a$ is an affine mapping from Euclidean space $\Lambda$ to $E$, and

$$
\Xi=\{\xi: A \xi+a \in \mathcal{X}\} .
$$

Then vector field $\Phi(\xi)=A^{T} F(A \xi+a): \Xi \rightarrow \Lambda$ is continuous and monotone, and the conic constraint

$$
\tau=t-\langle g, a\rangle, \gamma=A^{T} g, X(A \xi+a)+G g+t T+U u \leq_{\mathbf{K}} a
$$

in variables $\tau, \gamma, \xi, g, t$, and $u$ represents $(\Phi, \Xi)$.

When (32) nearly represents $(F, \mathcal{X})$, conic constraint (33) nearly represents $(\Phi, \Xi)$.

\subsection{Illustrations}

\subsection{1 "Academic" illustration}

Let $\mathcal{K}$ contain Lorentz cones, $M \in \mathbf{R}^{n \times n}$ be such that $\bar{M}=\frac{1}{2}\left[M+M^{T}\right] \succeq 0$, and let $\mathcal{X} \subset \mathbf{R}_{+}^{n}$ be nonempty and $\mathcal{K}$-representable:

$$
\mathcal{X}=\left\{x: \exists u_{0}: A_{0} x+B_{0} u_{0} \leq_{\mathbf{K}_{0}} a_{0}\right\}
$$

Suppose that the operator

$$
F(x)=M x-\left[f_{1}(x) ; \ldots ; f_{n}(x)\right]
$$

is monotone on $\mathcal{X}$, and that functions $f_{i}(x)$ and $-\sum_{i} x_{i} f_{i}(x)$ are $\mathcal{K}$-representable on $\mathcal{X}$ :

$$
\begin{aligned}
& \left\{(x, s): x \in \mathcal{X}, s \geq f_{i}(x)\right\}=\left\{(x, s): \exists u_{1 i}: A_{1 i} x+s B_{1 i}+C_{1 i} u_{1 i} \leq_{\mathbf{K}_{1 i}} a_{1 i}\right\} \\
& \left\{(x, s): x \in \mathcal{X}, s \geq-\sum_{i} x_{i} f_{i}(x)\right\}=\left\{(x, s): \exists u_{2}: A_{2} x+s B_{2}+C_{2} u_{2} \leq_{\mathbf{K}_{2}} a_{2}\right\}
\end{aligned}
$$

Observe, that when $M \succeq \alpha I_{m}$ with $\alpha>0, F$ definitely is monotone provided that $f(x):=\left[f_{1}(x) ; \ldots ; f_{n}(x)\right]$ is Lipschitz continuous on $\mathcal{X}$ with Lipschitz constant w.r.t. $\|\cdot\|_{2}$ bounded with $\alpha$.

Given $x \in \mathcal{X}$ and $g \in \mathbf{R}^{n}$, let us consider the function

$$
f_{x, g}(y)=g^{T} y+F^{T}(y)(x-y)=-y^{T} \bar{M} y+\sum_{i}\left[g_{i} y_{i}+x_{i}\left[[M y]_{i}-f_{i}(y)\right]+y_{i} f_{i}(y)\right]: \mathcal{X} \rightarrow \mathbf{R} .
$$

We are in the situation where $x_{i} \geq 0$ for $x \in \mathcal{X}$, so that

$$
\begin{aligned}
& \left\{(y, r): y \in \mathcal{X}, f_{x, g}(y) \geq r\right\} \\
& =\left\{\begin{array}{ll}
(y, r): \exists u_{0}, s_{0}, s_{1 i}, s_{2}: & A_{0} y+B_{0} u_{0} \leq \mathbf{K}_{0} a_{0}, y^{T} \bar{M} y-s_{0} \leq 0 \\
& \sum_{i}\left[g_{i}+\left[M^{T} x\right]_{i}\right] y_{i}-s_{0}+\sum_{i} x_{i} s_{1 i}+s_{2} \geq r
\end{array}\right\} \\
& =\left\{(y, r): \exists u_{0}, s_{0}, s_{1 i}, s_{2}, u_{1 i}, u_{2}:\right. \\
& A_{0} y+B_{0} u_{0} \leq_{\mathbf{K}_{0}} a_{0}, y^{T} \bar{M} y-s_{0} \leq 0, A_{2} y-s_{2} B_{2}+C_{2} u_{2} \leq_{\mathbf{K}_{2}} a_{2} \\
& A_{1 i} y-s_{1 i} B_{1 i}+C_{1 i} u_{1 i} \leq_{\mathbf{K}_{1 i}} a_{1 i}, 1 \leq i \leq n \\
& \sum_{i}\left[g_{i}+\left[M^{T} x\right]_{i}\right] y_{i}-s_{0}+\sum_{i} x_{i} s_{1 i}+s_{2} \geq r
\end{aligned}
$$

Taking into account that $\bar{M} \succeq 0$, the constraint $y^{T} \bar{M} y-s_{0} \leq 0$ can be represented by strictly feasible conic constraint

$$
A_{3} y+s_{0} B_{3} \leq_{\mathbf{K}_{3}} a_{3}
$$

on Lorentz cone $\mathbf{L}^{3}$, we get

$$
\begin{aligned}
& \max _{y \in \mathcal{X}} f_{x, g}(y)=\sup _{y, u_{0}, s_{0}, s_{1 i}, s_{2}, u_{1 i}, u_{2}}\left\{\sum_{i}\left[g_{i}+\left[M^{T} x\right]_{i}\right] y_{i}-s_{0}+\sum_{i} x_{i} s_{1 i}+s_{2}:\right. \\
& \left.\begin{array}{l}
A_{0} y+B_{0} u_{0} \leq_{\mathbf{K}_{0}} a_{0}, A_{3} y+s_{0} B_{3} \leq \mathbf{K}_{3} a_{3} \\
A_{1 i} y-s_{1 i} B_{1 i}+C_{1 i} u_{1 i} \leq \mathbf{K}_{1 i} a_{1 i}, 1 \leq i \leq n, A_{2} y-s_{2} B_{2}+C_{2} u_{2} \leq_{\mathbf{K}_{2 i}} a_{2}
\end{array}\right\} .
\end{aligned}
$$


Assume that the system of conic constraints (37) in variables $y, s_{0}, u_{0}, s_{1 i}, s_{2}, u_{1 i}$ and $u_{2}$ is essentially strictly feasible. Then, by conic quality,

$$
\left.\begin{array}{rl}
\max _{y \in \mathcal{X}} f_{x, g}(y)=\min _{\mu, \nu, \xi_{i}, \eta}\left\{a_{0}^{T} \mu+a_{3}^{T} \nu+\sum_{i} a_{1 i}^{T} \xi_{i}+a_{2}^{T} \eta:\right. \\
M^{T} x+g=A_{0}^{T} \mu+A_{3}^{T} \nu+\sum_{i} A_{1 i}^{T} \xi_{i}+A_{2}^{T} \eta \\
x_{i}+B_{1 i}^{T} \xi_{i}=0,1 \leq i \leq n, B_{2}^{T} \eta=-1, B_{3}^{T} \nu=-1 \\
B_{0}^{T} \mu=0, C_{1 i}^{T} \xi_{i}=0,1 \leq i \leq n, C_{2}^{T} \eta=0, \\
\mu \geq \mathbf{K}_{0}^{*} 0, \nu \geq \mathbf{K}_{3}^{*} 0, \xi_{i} \geq_{\mathbf{K}_{1 i}^{*}} 0,1 \leq i \leq n, \eta \geq \mathbf{K}_{2}^{*} 0
\end{array}\right\} .
$$

Now, recalling what $f_{x, g}(y)$ is, we end up with $\mathcal{K}$-representation of $\mathcal{F}[F, \mathcal{X}]$ :

$$
\left.\begin{array}{rl}
\mathcal{F}[F, \mathcal{X}]:= & \left\{[t ; g ; x]: x \in \mathcal{X}, t-g^{T} y \geq F^{T}(y)[x-y] \forall y \in \mathcal{X}\right\} \\
= & \{t ; g ; x]: \exists u_{0}, \mu, \nu, \xi_{i}, \eta: \\
& t \geq a_{0}^{T} \mu+a_{3}^{T} \nu+\sum_{i} a_{1}^{T} \xi_{i}+a_{2}^{T} \eta, A_{0} x+B_{0} u_{0} \leq \mathbf{K}_{0} a_{0} \\
& M^{T} x+g=A_{0}^{T} \mu+A_{3}^{T} \nu+\sum_{i} A_{1 i}^{T} \xi_{i}+A_{2}^{T} \eta \\
& x_{i}+B_{1 i}^{T} \xi_{i}=0,1 \leq i \leq n, B_{2}^{T} \eta=-1, B_{3}^{T} \nu=-1 \\
& B_{0}^{T} \mu=0, C_{1 i}^{T} \xi_{i}=0,1 \leq i \leq n, C_{2}^{T} \eta=0 \\
& \mu \geq \mathbf{K}_{0}^{*} 0, \nu \geq \mathbf{K}_{3}^{*} 0, \xi_{i} \geq \mathbf{K}_{1 i}^{*} 0,1 \leq i \leq n, \eta \geq \mathbf{K}_{2}^{*} 0
\end{array}\right\},
$$

so that the conic constraint

$$
\left[\begin{array}{l}
t \geq a_{0}^{T} \mu+a_{3}^{T} \nu+\sum_{i} a_{1 i}^{T} \xi_{i}+a_{2}^{T} \eta, A_{0} x+B_{0} u_{0} \leq_{\mathbf{K}_{0}} a_{0} \\
x_{i}+B_{1 i}^{T} \xi_{i}=0,1 \leq i \leq n, B_{2}^{T} \eta=-1,, B_{3}^{T} \nu=-1 \\
B_{0}^{T} \mu=0, C_{1 i}^{T} \xi_{i}=0,1 \leq i \leq n, C_{2}^{T} \eta=0 \\
\mu \geq \mathbf{K}_{0}^{*} 0, \nu \geq \mathbf{K}_{3}^{*} 0, \xi_{i} \geq \mathbf{K}_{1 i}^{*} 0,1 \leq i \leq n, \eta \geq \mathbf{K}_{2}^{*} 0
\end{array}\right]
$$

in variables $t, g, x, u_{0}, \mu, \nu, \xi_{i}$ and $\eta$ is a $\mathcal{K}$-representation of $(F, \mathcal{X})$.

\subsubsection{Nash Equilibrium}

The "covering story" for this example is as follows:

$n \geq 2$ retailers intend to enter certain market, say, one of red herrings. To this end they should select their "selling capacities" (say, rent areas at malls) $x_{i}, 1 \leq i \leq n$, in given ranges $\mathcal{X}_{i}=\left[0, X_{i}\right]$ $\left(0<X_{i}<\infty\right)$. With the selections $x=\left[x_{1} ; \ldots ; x_{n}\right] \in \mathcal{X}=\mathcal{X}_{1} \times \ldots, \times \mathcal{X}_{n} \subset \mathbf{R}_{+}^{n}$, the monthly loss of the $i$-th retailer is

$$
\phi_{i}(x)=c_{i} x_{i}-\frac{x_{i}}{\sum_{j=1}^{K} x_{j}+a} b
$$

where $c_{i} x_{i}, c_{i}>0$, is the price of the capacity $x_{i}, b>0$ is the dollar value of the demand on red herrings, and $a>0$ is the total selling capacity of the already existing retailers; the term $-\frac{x_{i}}{\sum_{j=1}^{n} x_{j}+a} b$ is the minus the revenue of the $i$-th retailer under the assumption that the total demand is split between retailers proportionally to their selling capacities.

We want to find is a Nash equilibrium - a point $x^{*}=\left[x_{1}^{*} ; \ldots ; x_{n}^{*}\right] \in \mathcal{X}$ such that every one of the functions $\phi_{i}\left(x_{i}^{*}, \ldots, x_{i-1}^{*}, x_{i}, x_{i+1}^{*}, \ldots, x_{n}^{*}\right)$ attains its minimum over $x_{i} \in \mathcal{X}_{i}$ at $x_{i}=x_{i}^{*}$, so that for the $i$-th retailer there is no incentive to deviate from capacity $x_{i}^{*}$ provided that all other retailers $j$ stick to capacities $x_{j}^{*}$, and this is so for all $i$.

As is immediately seen, the Nash Equilibrium problem in question is convex, meaning that $\phi_{i}(x)$ are convex in $x_{i}$ and concave in $x^{i}=\left(x_{i}, \ldots, x_{i-1}, x_{i+1}, \ldots, x_{n}\right)$ and, on the top of it, $\sum_{i} \phi_{i}(x)$ is convex on $\mathcal{X}$. It is well known (for justification, see, e.g., [14) that for such a problem, the vector field

$$
F(x)=\left[\frac{\partial \phi_{1}(x)}{\partial x_{1}} ; \frac{\partial \phi_{2}(x)}{\partial x_{2}} ; \ldots ; \frac{\partial \phi_{n}(x)}{\partial x_{n}}\right]: \mathcal{X} \rightarrow \mathbf{R}^{n}
$$


is monotone, and that Nash Equilibria are exactly the weak=strong solutions to $\operatorname{VI}(F, \mathcal{X})$. Specifying $\mathcal{K}$ as the family of direct products of Lorentz cones, we are about to demonstrate that $F$ admits an explicit $\mathcal{K}$-representation on $\mathcal{X}$, allowing to reduce the problem of finding Nash Equilibrium to an explicit Second Order conic problem. This is how the construction goes (to save notation, in what follows we set $b=a=1$, which always can be achieved by appropriate scaling of the capacities and loss functions):

1. Observe that all we need is a $\mathcal{K}$-representation $\mathcal{R}(t, g, x, u)$ of $(F, \mathcal{X})$ - a $\mathcal{K}$-conic constraint in variables $t \in \mathbf{R}, g \in \mathbf{R}^{n}, x \in \mathbf{R}^{n}$ and additional variables $u$-satisfying the requirements specified in Section 4.2.1 By Proposition 4.1, given $\mathcal{R}$, for $\epsilon \geq 0$ we can write down an explicit $\mathcal{K}$-conic constraint $\mathcal{C}_{\epsilon}(t, g, x, u, v)$ in variables $t, g, x$ and additional variables $u, v$, with the size of the constraint (dimension of the associated cone and the total number of variables) independent of $\epsilon$ such that the constraint is feasible, and the $x$-component of any feasible solution to the constraint is an $\epsilon$-solution to $\operatorname{VI}(F, \mathcal{X})$ :

$$
x \in \mathcal{X} \& \epsilon_{\mathrm{VI}}[x \mid F]=\max _{y \in \mathcal{X}} F^{T}(y)[x-y] \leq \epsilon .
$$

As a result, finding $\epsilon$-solution to $\mathrm{VI}(F, \mathcal{X})$ reduces to solving an explicit solvable Second Order feasibility conic problem of the size independent of $\epsilon$.

2. Thus, all we need is to find an explicit $\mathcal{K}$-representation of $(F, \mathcal{X})$. This can be done as follows:

(a) Consider the convex-concave function

$$
\psi(u, v)=-\frac{u}{u+v+1}: \mathbf{R}_{+}^{2} \rightarrow \mathbf{R}
$$

along with the associated monotone vector field

$$
\Phi(u, v)=\left[\frac{\partial \psi(u, v)}{\partial u} ;-\frac{\partial \psi(u, v)}{\partial v}\right]=\left[-\frac{v+1}{(u+v+1)^{2}} ;-\frac{u}{(u+v+1)^{2}}\right] .
$$

Let also $A_{i}$ be $2 \times n$ matrix with $i$-th column $[1 ; 0]$ and all remaining columns equal to [0;1], and let $G(x)=\nabla\left(\frac{1}{\sum_{j} x_{j}+1}\right)$. As is immediately seen, we have

$$
\forall x \in \mathbf{R}_{+}^{n}: \Psi(x):=2 c+\sum_{i=1}^{n} A_{i}^{T} \Phi\left(A_{i} x\right)+G(x)=2 F(x),
$$

so that a $\mathcal{K}$-representation of $F$ is readily given by $\mathcal{K}$-representations of the constant monotone vector field $\equiv c$, of $\Phi$, and of $G$ via our calculus (rules on affine substitution of argument and on summation).

A $\mathcal{K}$-representation of the constant vector field $\equiv c$ is trivial; it is given by the system of linear equalities $t=c^{T} x, g=c$ on variables $(t, g, x) \in \mathbf{R} \times \mathbf{R}^{n} \times \mathbf{R}^{n}$. A $\mathcal{K}$-representation of the gradient vector field $G(x)$ is given by calculus rule 2 in Section 4.3.1, we are in the case where (18) reads

$$
\begin{aligned}
\{(x, s): s \geq f(x), x \in X\}=\left\{\begin{array}{l}
(x):-x \leq 0, x \leq X:=\left[X_{1} ; \ldots ; X_{n}\right], \\
\end{array}\right. & \underbrace{-\left[0 ; \sum_{i} x_{i}-s ; \sum_{i} x_{i}+s\right] \leq_{\mathbf{L}^{3}}[2 ; 1 ; 1]}\}, \\
& \Leftrightarrow \quad \begin{array}{l}
\quad\left(\sum_{i} x_{i}+1+s\right)^{2} \geq\left(\sum_{i} x_{i}+1-s\right)^{2}+4 \\
s\left(\sum_{i} x_{i}+1\right) \geq 1
\end{array}
\end{aligned}
$$

so that (19), as seen from immediate computation, reduces to the system of constraints

$$
\begin{gathered}
t \geq r+s, 0 \leq x \leq X, r \geq \sum_{i} \max \left[X_{i}\left(g_{i}+\theta\right), 0\right]+\theta-2 \nu \\
\underbrace{\left[2 ; \sum_{i} x_{i}+1-s ; \sum_{i} x_{i}+1+s\right] \geq_{\mathbf{L}^{3}} 0}_{\Leftrightarrow s\left(\sum_{j} x_{j}+1\right) \geq 1}, \underbrace{[2 \nu ; \theta-1 ; \theta+1] \geq_{\mathbf{L}^{3}} 0}_{\Leftrightarrow \nu^{2} \leq \theta}
\end{gathered}
$$

in variables $t, g, x, \theta, \nu$. 
(b) It remains to find a $\mathcal{K}$-representation of the vector field $\Phi$ on a rectangle $\Xi=\mathcal{U} \times \mathcal{V}, \mathcal{U}=[0, U]$, $\mathcal{V}=[0, V]$ with given $U>0, V>0$ (they should be large enough in order for $A_{i} x, x \in \mathcal{X}$, to take values in the rectangle).

Let us use the construction described in item 3 of Section 4.3.1. By (i) of item 3, the set

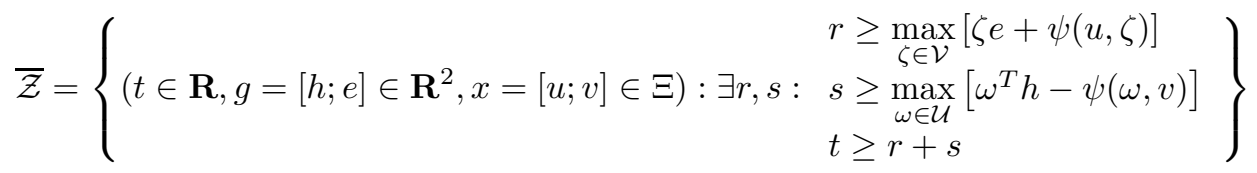

satisfies the relations

$$
\begin{array}{rll}
\overline{\mathcal{Z}} & \subset & \mathcal{F}[\Phi, \Xi] \\
x \in \mathcal{X}, g=\Phi(x), t \geq x^{T} \Phi(x) & \Rightarrow & {[t ; g ; x] \in \overline{\mathcal{Z}},}
\end{array}
$$

so that all we need to get a $\mathcal{K}$-representation of $\Phi$ is to represent $\overline{\mathcal{Z}}$ as a projection onto the plane of $t, g, x$-variables of the solution set of $\mathcal{K}$-conic constraint in variables $t, g, x$ and additional variables. To this end note that for all $(u, v) \in \Xi=\mathcal{U} \times \mathcal{V}$ one has

$$
\psi(u, v)=\min _{f, t, s, \tau}\{f v+t: \underbrace{\left[\begin{array}{l}
\tau \geq 0,1 \geq f \geq 0, s \geq 0, t \leq 1, s+\tau \leq 1 \\
{[2 s ; u-f ; u+f] \in \mathbf{L}^{3}} \\
{[2(1-s) ; t-f ; t-f+2] \in \mathbf{L}^{3}} \\
{[2 ; u-\tau+1 ; u+\tau+1] \in \mathbf{L}^{3}}
\end{array}\right]}_{\Leftrightarrow(f, t, s, \tau) \in \Pi_{+}}\}
$$

and

$$
-\psi(u, v)=\min _{f, t, s}\{f u+t: \underbrace{\left[\begin{array}{l}
1 \geq f \geq 0,0 \leq s \leq 1, t \leq 1 \\
{[2 s ; v+1-f ; v+1+f] \in \mathbf{L}^{3}} \\
{[2(1-s) ; t-1 ; t+1] \in \mathbf{L}^{3}}
\end{array}\right]}_{\Leftrightarrow(f, t, s) \in \Pi_{-}}\},
$$

see Section $B$ for justification. Since $\Pi_{ \pm}$are nonempty convex compact sets, by the Sion-Kakutani Theorem one has from (38):

$$
\begin{aligned}
\max _{\zeta \in \mathcal{V}}[\zeta e+\psi(u, \zeta)] & =\max _{0 \leq \zeta \leq V} \min _{f, t, s, \tau}\left\{[f+e] \zeta+t:(f, t, s, \tau) \in \Pi_{+}\right\} \\
& =\min _{f, t, s, \tau}\left\{\max _{0 \leq \zeta \leq V}[f+e] \zeta+t:(f, t, s, \tau) \in \Pi_{+}\right\} \\
& =\min _{f, t, s, \tau}\left\{\max [0, V(f+e)]+t:(f, t, s, \tau) \in \Pi_{+}\right\},
\end{aligned}
$$

and from (39):

$$
\begin{aligned}
\max _{\omega \in \mathcal{U}}[\omega h-\psi(\omega, v)] & =\max _{0 \leq \omega \leq U} \min _{f, t, s}\left\{[f+h] \omega+t:(f, t, s) \in \Pi_{-}\right\} \\
& =\min _{f, t, s}\left\{\max _{0 \leq \omega \leq U}[f+h] \omega+t:(f, t, s) \in \Pi_{-}\right\} \\
& =\min _{f, t, s}\left\{\max [0, U(f+h)]+t:(f, t, s) \in \Pi_{-}\right\} .
\end{aligned}
$$

As a result,

$$
\begin{aligned}
\overline{\mathcal{Z}}=\{ & \left(t \in \mathbf{R}, g=[h ; e] \in \mathbf{R}^{2}, 0 \leq x:=[u ; v] \leq[U ; V]\right): \exists r, s, f_{ \pm}, t_{ \pm}, s_{ \pm}, \tau: \\
& \left.t \geq r+s, r \geq \max \left[0, V\left(f_{+}+e\right)\right]+t_{+}, s \geq \max \left[0, U\left(f_{-}+h\right)\right]+t_{-}\right\} . \\
& \left(f_{+}, t_{+}, s_{+}, \tau\right) \in \Pi_{+},\left(f_{-}, t_{-}, s_{-}\right) \in \Pi_{-}
\end{aligned}
$$

Recalling what are $\Pi_{ \pm}$, we can straightforwardly represent $\overline{\mathcal{Z}}$ as projection onto the space of $t, g, x$-variables of a set given by a $\mathcal{K}$-conic inequality, ending up with an explicit $\mathcal{K}$-representation of $\Phi$. 
Remark 4.2 The just outlined construction can be used in the case of a general convex Nash Equilibrium problem where, given $n$ convex compact sets $\mathcal{X}_{i} \subset \mathbf{R}^{k_{i}}$ and $n$ continuously differentiable functions $\phi_{i}\left(x_{1}, \ldots, x_{n}\right): \mathcal{X}:=\mathcal{X}_{1} \times \ldots \times \mathcal{X}_{n} \rightarrow \mathbf{R}$ with $\phi_{i}$ convex in $x_{i}$, concave in $\left(x_{1}, \ldots, x_{i-1}, x_{i+1}, \ldots, x_{n}\right)$ and such that $\sum_{i} \phi_{i}$ is convex, one is looking for Nash Equilibria-points $x^{*}=\left[x_{1}^{*} ; \ldots ; x_{n}^{*}\right] \in \mathcal{X}$ such that for every $i$, the function $\phi_{i}\left(x_{1}^{*}, \ldots, x_{i-1}^{*}, x_{i}, x_{i+1}^{*}, \ldots, x_{n}^{*}\right)$ attains its minimum over $x_{i} \in \mathcal{X}_{i}$ at $x_{i}=x_{i}^{*}$. As it was already mentioned, these equilibria are exactly the weak=strong solutions to $\operatorname{VI}(F, \mathcal{X})$ where the monotone vector field $F$ is given by $F\left(x_{1}, \ldots, x_{n}\right)=\left[\frac{\partial \phi_{1}(x)}{\partial x_{1}} ; \ldots ; \frac{\partial \phi_{n}(x)}{\partial x_{n}}\right]$. Observing that the monotone vector fields

$$
F^{i}(x)=\left[-\frac{\partial \phi_{i}(x)}{\partial x_{1}} ; \ldots ;-\frac{\partial \phi_{i}(x)}{\partial x_{i-1}} ; \frac{\partial \phi_{i}(x)}{\partial x_{i}} ;-\frac{\partial \phi_{i}(x)}{\partial x_{i+1}} ; \ldots ;-\frac{\partial \phi_{i}(x)}{\partial x_{n}}\right]
$$

associated with convex-concave functions $\phi_{i}$ and the monotone vector field $F^{0}(x)=\nabla\left(\sum_{i} \phi_{i}(x)\right)$ are linked to $F$ by the relation $2 F=\sum_{i=0}^{n} F^{i}$, we see that in order to get a $\mathcal{K}$-representation of $F$, it suffices to have at our disposal $\mathcal{K}$-representations of $F^{i}, 0 \leq i \leq n$. These latter representations, in good cases, can be built according to recipes presented in items 2 and 3 of Section 4.3.1.

The latter remark puts in proper perspective the "red herring" illustration which by itself is of no actual interest: setting $s=\sum_{i} x_{i}+1, x \in \mathcal{X}$ is a Nash Equilibrium if and only if

$$
c_{i}-\frac{1}{s}+\frac{x_{i}}{s^{2}}=\frac{\partial \phi_{i}(x)}{\partial x_{i}} \begin{cases}\geq 0, & x_{i}=0 \\ =0, & 0<x_{i}<X_{i} \\ \leq 0, & x_{i}=X_{i} .\end{cases}
$$

As a result, finding the equilibrium reduces to solving on the segment $s \in\left[1, \sum_{i} X_{i}+1\right]$ the univariate equation

$$
\sum_{i} x_{i}(s)+1=s, x_{i}(s)= \begin{cases}0, & s\left(1-s c_{i}\right)<0 \\ s\left(1-s c_{i}\right), & 0 \leq s\left(1-s c_{i}\right) \leq X_{i} \\ X_{i}, & s\left(1-s c_{i}\right)>X_{i} .\end{cases}
$$

\section{Acknowledgment}

This work was supported by Multidisciplinary Institute in Artificial intelligence MIAI @ Grenoble Alpes (ANR-19-P3IA-0003).

\section{References}

[1] Aghassia, M., Bertsimas. D., Perakis, G. "Solving asymmetric variational inequalities via convex optimization" - Operations Research Letters 34 (2006) 481-490

[2] Andersen, E. D. MOSEK optimization suite, MOSEK ApS, 2019.https://docs.mosek.com/9.2/intro.pdf

[3] Boyd, S., Vandenberghe, L. Convex Optimization, Cambridge University Press, 2004. https://web.stanford.edu/ boyd/cvxbook/bv_cvxbook.pdf

[4] Ben-Tal, A., Nemirovski, A. Lectures on Modern Convex Optimization: Analysis, Algorithms and Engineering Applications, MPS-SIAM Series on Optimization, SIAM, Philadelphia, 2001.

[5] Ben-Tal, A., Nemirovski, A. Lectures on Modern Convex Optimization [ISyE GaTech], http://www2.isye.gatech.edu/ nemirovs/LMCOLN2021WithSol.pdf

[6] Ghaoui, L., Oks, M., Oustry, F. Worst-case value-at-risk and robust portfolio optimization: A conic programming approach - Operations research, 51(4) 2003, 543-556.

[7] Facchinei, F., Pang, J.-S. Finite Dimensional Variational Inequalities and Complementarity Problems, Vol. 1 and 2, Springer, 2003. 
[8] Goldfarb, D., Iyengar, G. Robust portfolio selection problems - Mathematics of operations research, 28(1) 2003, 1-38.

[9] Grant, M., and Boyd, S. CVX: Matlab software for disciplined convex programming, version 2.2, CVX Research, Inc, 2020. http://cvxr.com/cvx/doc/

[10] Juditsky, A., Nemirovski, A. Statistical Inference via Convex Optimization, Princeton University Press, 2020. http://www2.isye.gatech.edu/ nemirovs/StatOptPUPWeb.pdf

[11] Kojima, M., Megiddo, N., Noma, T., Yoshise, A. A Unified Approach to Interior Point Algorithms for Linear Complementarity Problems, Lecture Notes in Computer Science 538, Springer, 1991.

[12] Monteiro, R.D.C., Zanjacomo, P. "General Interior-Point Maps and Existence of Weighted Paths for Nonlinear Semidefinite Complementarity Problems" - Mathematics of Operations Research 252000 , 381-399.

[13] Nesterov, Yu., Nemirovskii, A. Interior-Point Polynomial Algorithms in Convex Programming, SIAM, 1994.

[14] Nemirovski, A., Onn, S., Rothblum, U. "Accuracy certificates for computational problems with convex structure" - Mathematics of Operations Research 35:1 2010, 52-78. https://www2.isye.gatech.edu/ nemirovs/MOR_AccuracyCertificates.pdf

[15] Nesterov, Yu. "Squared Functional Systems and Optimization Problems" - In: Frenk, H., Roos, C., Terlaky, T., Zhang. S. (Eds.) High Performance Optimization, Springer 2000, 405-440.

[16] Petra C. G., Potra F. A. "A homogeneous model for monotone mixed horizontal linear complementarity problems" - Computational Optimization and Applications 72 2019, 241-267.

[17] Ralph, D., Wright, S. J. "Superlinear convergence of an interior-point method for monotone variational inequalities" - In Ferris, M. C., Pang, J. S. (Eds.) Complementarity and Variational Problems: State of the Art, SIAM Publications, Philadelphia, 1997, 345-385.

[18] Sim, C. K. "Interior point method on semi-definite linear complementarity problems using the Nesterov-Todd (NT) search direction: polynomial complexity and local convergence" - Computational Optimization and Applications $\mathbf{7 4}$ 2019, 583-621.

[19] Toh, K., Todd, M., Tütüncü, R. H. On the implementation and usage of SDPT3 - a Matlab software package for semidefinite-quadratic-linear programming, version 4.0 - In Anjos, M. F., Lasserre, J. B. (Eds.) Handbook on semidefinite, conic and polynomial optimization, Springer, Boston, MA, 2012, 715754 .

\section{A Derivations for Section 4.3}

\section{A.1 Verification of "raw materials"}

1. [Affine monotone vector field] Note first that the symmetric part $\bar{A}$ of $A$ is $\succeq 0$ due to the monotonicity of $F$ on $E$, so that (17) can be rewritten as a conic constraint on a direct product of properly selected Lorentz cone and nonnegative orthant, and this direct product belongs to $\mathcal{K}$. Second, when $t, g, x$ solve (17), for every $y \in E$ one has $x^{T} A x=x^{T} \bar{A} x$, whence

$$
t-y^{T} g=t-y^{T}[A x+a]=\underbrace{t-x^{T}[A x+a]}_{=t-x^{T} \bar{A} x-x^{T} a \geq 0}-[y-x]^{T}[A x+a] \geq[x-y]^{T}[A x+a] \geq[x-y]^{T}[A y+a],
$$

that is, $[t ; g ; x] \in \mathcal{F}[F, E]$, as required in item (i) of a representation of $(F, E)$. Furthermore, when $x \in E$, setting $g=F(x)=A x+a$ and $t=x^{T} F(x)=x^{T}[A x+a]=x^{T}[\bar{A} x+a]$, we see that $[t ; g ; x]$ satisfy (17), as required in item (ii) of a representation of $(F, E)$. 
2. [Gradient field of continuously differentiable $\mathcal{K}$-representable convex function] Let

$$
f_{*}(y)=\sup _{x \in \mathcal{X}}\left[x^{T} y-f(x)\right]
$$

be the Fenchel transform of $f$ :

$$
\begin{aligned}
f_{*}(y) & =\sup _{x \in \mathcal{X}}\left[x^{T} y-f(x)\right]=\sup _{x \in \mathcal{X}, s \geq f(x)}\left[x^{T} y-s\right] \\
& =\sup _{x, u}\left[x^{T} y-s: A x+s p+Q u \leq \mathbf{K} a\right] \\
& =\min _{\lambda}\left\{a^{T} \lambda: A^{T} \lambda=y, p^{T} \lambda=-1, Q^{T} \lambda=0, \lambda \geq_{\mathbf{K}^{*}} 0\right\} \quad \text { [by conic duality], }
\end{aligned}
$$

that is,

$$
r \geq f_{*}(g) \Leftrightarrow \exists \lambda: r \geq a^{T} \lambda, A^{T} \lambda=g, p^{T} \lambda=-1, Q^{T} \lambda=0, \lambda \geq \mathbf{K}^{*} 0 .
$$

Next, let

$$
\begin{aligned}
\mathcal{Z} & =\left\{(t, g, x): \exists s, r: s \geq f(x), r \geq f_{*}(g), s+r \leq t\right\} \\
& =\left\{(t, g, x): \exists s, r, u, \lambda: \begin{array}{l}
t \geq s+r, A x+s p+Q u \leq_{\mathbf{K}} a \\
r \geq a^{T} \lambda, A^{T} \lambda=g, p^{T} \lambda=-1, Q^{T} \lambda=0, \lambda \geq_{\mathbf{K}^{*}} 0
\end{array}\right\}
\end{aligned}
$$

where (41, b) is due to (18) and (40). Thus, $\mathcal{Z}$ is the projection of the solution set of (19) on the space of $t, g, x$-variables, and we should check that

(i) $\mathcal{Z} \subset \mathcal{F}\left[f^{\prime}(\cdot), \mathcal{X}\right]$, and

(ii) when $x \in \mathcal{X}, g=f^{\prime}(x)$, and $t=x^{T} f^{\prime}(x)$, we have $[t ; g ; x] \in \mathcal{Z}$.

(i): Let $(t, g, x) \in \mathcal{Z}$. We have $t \geq s+r$ for properly selected $s \geq f(x)$ and $r \geq f_{*}(g)$, whence for $y \in \mathcal{X}$ it holds

$$
t-g^{T} y \geq f(x)+f_{*}(g)-g^{T} y \geq f(x)-f(y) \geq(x-y)^{T} f^{\prime}(y),
$$

that is, $[t ; g ; x] \in \mathcal{F}\left[f^{\prime}(\cdot), \mathcal{X}\right]$. (i) is proved.

(ii): Given $x \in \mathcal{X}$, let us set $g=f^{\prime}(x), t=x^{T} g, s=f(x), r=f_{*}(g)$. We have

$$
r=f_{*}(g)=\sup _{x^{\prime}}\left\{g^{T} x^{\prime}-f\left(x^{\prime}\right): x^{\prime} \in \mathcal{X}\right\}=g^{T} x-f(x)
$$

(the concluding equality is due to $g=f^{\prime}(x)$ ); thus, $t=r+s$. Invoking (41) $a$ ), we conclude that $(t, g, x) \in \mathcal{Z}$. (ii) is proved.

3. [Monotone vector field associated with $\mathcal{K}$-representable convex-concave function $\psi$ ]

Verifying $(i)$ :

$\mathbf{1}^{o}$. Let us show (23) $\left.a\right)$. Let $(t, g=[h ; e], x=[u ; v]) \in \mathcal{Z}$, and let $r, s$ be reals which, taken together with $t, g, x$, form a feasible solution to the system of constraints in (22). For $y=[w ; z] \in \mathcal{X}$ we have

$$
\begin{aligned}
t-y^{T} g & \geq r+s-w^{T} h-z^{T} e[\text { by }[22 c)] \\
& \left.\geq\left[z^{T} e+\psi(u, z)\right]+\left[w^{T} h-\psi(w, v)\right]-w^{T} h-z^{T} e[\text { by [22 } a, b)\right] \\
& =\psi(u, z)-\psi(w, v)=[\psi(u, z)-\psi(w, z)]+[\psi(w, z)-\psi(w, v)] \\
& \geq[u-w]^{T} \nabla_{u} \psi(w, z)-[v-z]^{T} \nabla_{v} \psi(w, z)[\text { because } \psi \text { is convex-concave] } \\
& =[x-y]^{T} F(y)
\end{aligned}
$$

implying that $\mathcal{Z} \subset \mathcal{F}[F, \mathcal{X}]$.

$\mathbf{2}^{o}$. Let us now verify $\left.(22] b\right)$. Given $x=[u ; v] \in \mathcal{X}$, let us set

$$
g=F(x)=[h ; e], h=\nabla_{u} \psi(u, v), e=-\nabla_{v} \psi(u, v) ; \bar{t}=x^{T} F(x)=h^{T} u+e^{T} v,
$$

and let $t>\bar{t}$. The function $\zeta^{T} e+\psi(u, \zeta)$ of $\zeta \in \mathcal{V}$ is concave, and its gradient w.r.t. $\zeta$ taken at the point $\zeta=v$ vanishes, implying that

$$
\bar{r}:=v^{T} e+\psi(u, v) \geq \zeta^{T} e+\psi(u, \zeta) \forall \zeta \in \mathcal{V} .
$$


Similarly, the function $\omega^{T} h-\psi(\omega, v)$ of $\omega \in \mathcal{U}$ is concave. and its gradient w.r.t. $\omega$ taken at the point $\omega=u$ vanishes, implying that

$$
\bar{s}:=u^{T} h-\psi(u, v) \geq \omega^{T} h-\psi(\omega, v) \forall \omega \in \mathcal{U} .
$$

Observing that $\bar{r}+\bar{s}=\bar{t}<t$, we can find $r>\bar{r}$ and $s>\bar{s}$ in such a way that $r+s \leq t$. Looking at the constraints (22. $a-c)$, we conclude that whenever $x=[u ; v] \in \mathcal{X}$ and $t>x^{T} F(x)$, the triple $(t, g=F(x), x)$ can be augmented by $r, s$ to satisfy all constraints $(22 a-c)$, that is, $(t, g, x) \in \mathcal{Z}$, as claimed in $(23, b)$.

Verifying (ii): Clearly, all we need in order to justify claim in (ii) is to show that the set

$$
\mathcal{Z}^{+}=\left\{(g=[h ; e], x=[u ; v], r, s): u \in \mathcal{U}, v \in \mathcal{V}, r>\max _{z \in \mathcal{V}}\left[z^{T} e+\psi(u, z)\right], s>\max _{w \in \mathcal{U}}\left[w^{T} h-\psi(w, v)\right]\right\}
$$

is the projection of the solution set of the conic constraint

$$
\left[\begin{array}{l}
r \geq \theta+\gamma^{T} b+\tau, \eta \geq 0, \theta \geq 0, \eta \theta \geq 1 \\
s \geq \theta^{\prime}+c^{T} \delta+a^{T} \epsilon, \eta^{\prime} \geq 0, \theta^{\prime} \geq 0, \eta^{\prime} \theta^{\prime} \geq 1 \\
A u+B \alpha \leq_{\mathbf{K}} a, C v+D \beta \leq_{\mathbf{L}} b, P f+\tau p+Q \xi+R u \leq_{\mathbf{M}} c \\
C^{T} \gamma=f+e, D^{T} \gamma=0, \gamma \mathbf{L}^{*} 0, \delta \geq_{\mathbf{M}^{*}} 0, \epsilon \mathbf{K}_{\mathbf{K}^{*}} 0 \\
P^{T} \delta+v=0, p^{T} \delta=-1, Q^{T} \delta=0, R^{T} \delta+A^{T} \epsilon=h, B^{T} \epsilon=0
\end{array}\right]
$$

in variables $x=[u ; v], g=[h ; e], r, s, \alpha, \beta, \gamma, f, \tau, \xi, \delta, \epsilon, \eta, \theta, \eta^{\prime}$, and $\theta^{\prime}$ on the plane of $g=[h ; e], x=$ $[u ; v], r, s$-variables. Here is the proof.

$\mathbf{1}^{o}$. Recall that $\mathcal{V}$ is convex and compact. Thus, we have

$$
\begin{aligned}
& \forall(x=[u ; v] \in \mathcal{X}, g=[h ; e]): \\
& \max _{z \in \mathcal{V}}\left[z^{T} e+\psi(u, z)\right]=\max _{z \in \mathcal{V}}\left[z^{T} e+\inf _{f, \tau, \xi}\left[f^{T} z+\tau: P f+\tau p+Q \xi+R u \leq_{\mathbf{M}} c\right]\right] \text { [by (21)] } \\
& =\max _{z \in \mathcal{V}} \inf _{f, \tau, \xi}\left[(f+e)^{T} z+\tau: P f+\tau p+Q \xi+R u \leq_{\mathbf{M}} c\right] \\
& =\inf _{f, \tau, \xi}\left\{\max _{z \in \mathcal{V}}(f+e)^{T} z+\tau: P f+\tau p+Q \xi+R u \leq_{\mathbf{M}} c\right\} \text { [by the Sion-Kakutani Theorem] } \\
& \left.=\inf _{f, \tau, \xi}\left[\max _{z, \beta}\left[(f+e)^{T} z+\tau: C z+D \beta \leq_{\mathbf{L}} b\right]: P f+\tau p+Q \xi+R u \leq_{\mathbf{M}} c\right] \quad[\text { by (20, } b)\right] \\
& =\inf _{f, \tau, \xi, \gamma}\left\{\gamma^{T} b+\tau: C^{T} \gamma=f+e, D^{T} \gamma=0, \gamma \geq_{\mathbf{L}^{*}} 0, P f+\tau p+Q \xi+R u \leq_{\mathbf{M}} c\right\}
\end{aligned}
$$

[by conic duality; recall that $(20, b)$ is essentially strictly feasible].

Together with (20) the latter relation results in

$$
\begin{aligned}
& \left\{(x=[u ; v], g=[h ; e], r): x \in \mathcal{X}, r>\max _{z \in \mathcal{V}}\left[z^{T} e+\psi(u, z)\right]\right\} \\
& =\{(x=[u ; v], g=[h ; e], r): \quad \exists f, \tau, \xi, \alpha, \beta, \gamma: \\
& \left.\begin{array}{l}
r>\gamma^{T} b+\tau, A u+B \alpha \leq_{\mathbf{K}} a, C v+D \beta \leq_{\mathbf{L}} b, \gamma \geq_{\mathbf{L}^{*}} 0 \\
C^{T} \gamma=f+e, D^{T} \gamma=0, P f+\tau p+Q \xi+R u \leq_{\mathbf{M}} c
\end{array}\right\} \\
& =\{(x=[u ; v], g=[h ; e], r): \quad \exists f, \tau, \xi, \alpha, \beta, \gamma, \eta, \theta: \\
& r \geq \gamma^{T} b+\tau+\theta, \eta \geq 0, \theta \geq 0, \eta \theta \geq 1 \\
& \left.\begin{array}{l}
A u+B \alpha \leq_{\mathbf{K}} a, C v+D \beta \leq_{\mathbf{L}} b,, \gamma \geq_{\mathbf{L}^{*}}, \\
C^{T} \gamma=f+e, D^{T} \gamma=0, P f+\tau p+Q \xi+R u \leq_{\mathbf{M}} c
\end{array}\right\} .
\end{aligned}
$$


$\mathbf{2}^{o}$. When $v \in \mathcal{V}$ and $h \in \mathbf{R}^{n_{u}}$ we have

$$
\begin{aligned}
& \left.\max _{w \in \mathcal{U}}\left[w^{T} h-\psi(w, v)\right]=\max _{w \in \mathcal{U}}\left[w^{T} h+\sup _{f, \tau, \xi}\left[-f^{T} v-\tau: P f+\tau p+Q \xi+R w \leq_{\mathbf{M}} c\right]\right] \text { [by (21)] }\right] \\
& \left.=\sup _{w, f, \tau, \xi, \alpha}\left[w^{T} h-f^{T} v-\tau: P f+\tau p+Q \xi+R w \leq_{\mathbf{M}} c, A w+B \alpha \leq_{\mathbf{K}} a\right][\text { by (20] } a)\right] \\
& =\min _{\delta, \epsilon}\left\{c^{T} \delta+a^{T} \epsilon: \begin{array}{l}
P^{T} \delta+v=0, p^{T} \delta=-1, Q^{T} \delta=0, R^{T} \delta+A^{T} \epsilon=h \\
B^{T} \epsilon=0, \delta \geq \mathbf{M}^{*} 0, \epsilon \mathbf{K}^{*} 0
\end{array}\right. \\
& {\left[\begin{array}{l}
\text { by conic duality; recall that the } \mathcal{K} \text {-representations (20) } a) \\
\text { of } \mathcal{U} \text { and (21) of } \psi \text { are essentially strictly feasible. }
\end{array}\right]}
\end{aligned}
$$

Taken together with (20), the latter relation results in

$$
\begin{aligned}
& \left\{(x=[u ; v], g=[h ; e], s): x \in \mathcal{X}, s>\max _{w \in \mathcal{U}}\left[h^{T} w-\psi(w, u)\right]\right\} \\
& =\{(x=[u ; v], g=[h ; e], s): \exists \alpha, \beta, \delta, \epsilon: \\
& \left.\begin{array}{l}
s>c^{T} \delta+a^{T} \epsilon, A u+B \alpha \leq_{\mathbf{K}} a, C v+D \beta \leq_{\mathbf{L}} b, \delta \geq_{\mathbf{M}^{*} 0, \epsilon \geq \mathbf{K}^{*} 0} \\
P^{T} \delta+v=0, p^{T} \delta=-1, Q^{T} \delta=0, R^{T} \delta+A^{T} \epsilon=h, B^{T} \epsilon=0
\end{array}\right\} \\
& =\left\{(x=[u ; v], g=[h ; e], s): \exists \alpha, \beta, \delta, \epsilon, \eta^{\prime}, \theta^{\prime}:\right. \\
& s \geq \theta^{\prime}+c^{T} \delta+a^{T} \epsilon, \eta^{\prime} \geq 0, \theta^{\prime} \geq 0, \eta^{\prime} \theta^{\prime} \geq 1 \\
& \left.\begin{array}{l}
A u+B \alpha \leq_{\mathbf{K}} a, C v+D \beta \leq_{\mathbf{L}} b, \delta \geq_{\mathbf{M}^{*}} 0, \epsilon \geq_{\mathbf{K}^{*}} 0 \\
P^{T} \delta+v=0, p^{T} \delta=-1, Q^{T} \delta=0, R^{T} \delta+A^{T} \epsilon=h, B^{T} \epsilon=0
\end{array}\right\} .
\end{aligned}
$$

Finally, (43) and (44) together imply that

$$
\begin{aligned}
& \mathcal{Z}^{+}:=\left\{(g=[h ; e], x=[u ; v], r, s): u \in \mathcal{U}, v \in \mathcal{V}, r>\max _{z \in \mathcal{V}}\left[z^{T} e+\psi(u, z)\right], s>\max _{w \in \mathcal{U}}\left[w^{T} h-\psi(v, u)\right]\right\} \\
& =\left\{(g=[h ; e], x=[u ; v], r, s): \exists f, \tau, \xi, \alpha, \beta, \gamma, \eta, \theta, \delta, \epsilon, \eta^{\prime}, \theta^{\prime}:\right. \\
& r \geq \theta+\gamma^{T} b+\tau, \eta \geq 0, \theta \geq 0, \eta \theta \geq 1 \\
& s \geq \theta^{\prime}+c^{T} \delta+a^{T} \epsilon, \eta^{\prime} \geq 0, \theta^{\prime} \geq 0, \eta^{\prime} \theta^{\prime} \geq 1 \\
& \left.A u+B \alpha \leq_{\mathbf{K}} a, C v+D \beta \leq_{\mathbf{L}} b, P f+\tau p+Q \xi+R u \leq_{\mathbf{M}} c, \gamma \geq_{\mathbf{L}^{*}} 0, \delta \geq_{\mathbf{M}^{*}} 0, \epsilon \geq_{\mathbf{K}^{*}} 0\right\} \text {, } \\
& C^{T} \gamma=f+e, D^{T} \gamma=0, P^{T} \delta+v=0, p^{T} \delta=-1, Q^{T} \delta=0, R^{T} \delta+A^{T} \epsilon=h, B^{T} \epsilon=0
\end{aligned}
$$

as claimed in (42).

4. [Univariate monotone rational vector field] For evident reasons, it suffices to consider the case of $\mathcal{X}=[0,1]$; recall that $\beta(t)>0$ on $\mathcal{X}$. Let degrees of $\alpha$ and $\beta$ be $\mu$ and $\nu$, respectively, and let

$$
\kappa=\max [\mu, \nu]+1 .
$$

$\mathbf{1}^{0}$. Consider the curves

$$
\delta(t)=[t F(t) ; F(t) ; t]=\frac{1}{\beta(t)}[t \alpha(t) ; \alpha(t) ; t \beta(t)]:[0,1] \rightarrow \mathbf{R}^{3}, \gamma(t)=\frac{1}{\beta(t)}\left[1 ; t ; t^{2} ; \ldots ; t^{\kappa}\right]:[0,1] \rightarrow \mathbf{R}^{\kappa+1}
$$

For properly selected matrix $A$ we have

$$
\delta(t)=A \gamma(t), 0 \leq t \leq 1,
$$

whence

$$
\mathcal{Y}:=\operatorname{Conv}\{\delta(t), 0 \leq t \leq 1\}=A \mathcal{Z}, \mathcal{Z}=\operatorname{Conv}\{\gamma(t), 0 \leq t \leq 1\}
$$


We intend to build a semidefinite representation (SDR) of $\mathcal{Y}$ (i.e., $\mathcal{K}$-representation with $\mathcal{K}$ comprised of finite direct products of semidefinite cones). Semidefinite representability (and $\mathcal{K}$-representability in general) of a set is preserved when taking linear images: an SDR

$$
\mathcal{Z}=\{z: \exists u: \mathcal{A}(z, u) \succeq 0\} \quad[\mathcal{A}(z, u) \text { is affine in }[z ; u] \text { symmetric matrix }]
$$

of $\mathcal{Z}$ implies the representation

$$
\mathcal{Y}:=A \mathcal{Z}=\{y: \exists[z ; u]: y=A z, \mathcal{A}(z, u) \succeq 0\},
$$

and the system of the right hand side constraints can be written down as a Linear Matrix Inequality in variable $y$ and additional variables $z, u$. Thus, all we need is to build an SDR of $\mathcal{Z}$.

$\mathbf{2}^{0}$. We shall get SDR of $\mathcal{Z}$ from SDR of the "support cone"

$$
\mathcal{P}=\left\{[p ; q] \in \mathbf{R}^{\kappa+1} \times \mathbf{R}: \min _{z \in \mathcal{Z}} p^{T} z-q \geq 0\right\}=\left\{[p ; q]: \min _{0 \leq t \leq 1} p^{T} \gamma(t) \geq q\right\}
$$

of $\mathcal{Z}$.

Given $p=\left[p_{0} ; p_{1} ; \ldots ; p_{\kappa+1}\right] \in \mathbf{R}^{\kappa+1}$, let, with a slight abuse of notation, $p(t)=\sum_{i=0}^{\kappa} p_{i} t^{i}$ be the polynomial with coefficients $p_{i}, 0 \leq i \leq \kappa$. We have

$$
\begin{aligned}
& {[p ; q] \in \mathcal{P} \Leftrightarrow \frac{p(t)}{\beta(t)} \geq q \forall t \in[0,1]} \\
& \Leftrightarrow \frac{\left(1+\tau^{2}\right)^{\kappa} p\left(\tau^{2} /\left(1+\tau^{2}\right)\right)}{\left(1+\tau^{2}\right)^{\kappa} \beta\left(\tau^{2} /\left(1+\tau^{2}\right)\right)} \geq q \forall \tau \in \mathbf{R} \\
& \Leftrightarrow \underbrace{\left(1+\tau^{2}\right)^{\kappa} p\left(\tau^{2} /\left(1+\tau^{2}\right)\right)-q\left(1+\tau^{2}\right)^{\kappa} \beta\left(\tau^{2} /\left(1+\tau^{2}\right)\right)}_{=: \pi_{p, q}(\tau)} \geq 0 \forall \tau \in \mathbf{R} .
\end{aligned}
$$

Note that $\pi_{p, q}(\tau)$ is a polynomial of $\tau$ of degree $\leq 2 \kappa$, and the vector $\pi_{p, q}$ of coefficients of polynomial is linear in $[p ; q]: \pi_{p, q}=P[p ; q]$. We see that $\mathcal{P}$ is the inverse image of the cone $\mathcal{P}_{2 \kappa}$ of coefficients of polynomials of degree $\leq 2 \kappa$ which are nonnegative on the entire axis:

$$
\mathcal{P}=\left\{[p ; q]: P[p ; q] \in \mathcal{P}_{2 \kappa}\right\} .
$$

As was observed by Yu. Nesterov [15], the cone $\mathcal{P}_{2 \kappa}$ is the linear image of the semidefinite cone $\mathbf{S}_{+}^{\kappa+1}$ :

$$
\mathcal{P}_{2 \kappa}=\left\{\pi \in \mathbf{R}^{2 \kappa+1}: \exists x=\left[x_{i j}\right]_{0 \leq i, j \leq \kappa} \in \mathbf{S}_{+}^{\kappa+1}:[\mathcal{Q} x]_{\ell}:=\sum_{\substack{0 \leq i, j \leq \kappa, i+j=\ell}} x_{i j}=\pi_{\ell}, 0 \leq \ell \leq 2 \kappa\right\},
$$

and we arrive at a semidefinite representation of $\mathcal{P}$ :

$$
\mathcal{P}=\left\{[p ; q]: \exists x \in \mathbf{S}^{\kappa+1}: x \succeq 0 \& \mathcal{Q} x=P[p ; q]\right\} .
$$

We claim that this representation is essentially strictly feasible. Indeed, let $\bar{p}=[1 ; 1 ; \ldots ; 1] \in \mathbf{R}^{\kappa+1}$ and $\bar{q}=0$. Then

$$
\pi_{\bar{p}, \bar{q}}(\tau)=\left(1+\tau^{2}\right)^{\kappa}\left[1+\tau^{2} /\left(1+\tau^{2}\right)+\left[\tau^{2} /\left(1+\tau^{2}\right)\right]^{2}+\ldots+\left[\tau^{2} /\left(1+\tau^{2}\right)\right]^{\kappa}\right]=\sum_{i=0}^{\kappa} c_{i} \tau^{2 i}, c_{i}>0 \forall i,
$$

implying that with $\bar{x}=\operatorname{Diag}\left\{c_{0}, c_{1}, \ldots, c_{\kappa}\right\} \in \mathbf{S}^{\kappa+1}$ one has

$$
P[\bar{p} ; \bar{q}]=\mathcal{Q} \bar{x} \& \bar{x} \succ 0 .
$$

That is, $\bar{p}, \bar{q}$, and $\bar{x}$ satisfy all constraints in (45) and strictly satisfy the non-polyhedral constraint $x \succeq 0$, as required by essentially strict feasibility. 
$\mathbf{3}^{0}$. Now we are done: by its origin, $\mathcal{Z}$ is a convex compact set and as such is convex and closed, implying by duality that

$$
\begin{aligned}
\mathcal{Z}= & \left\{z \in \mathbf{R}^{\kappa+1}: p^{T} z-q \geq 0 \forall[p ; q] \in \mathcal{P}\right\} \\
= & \left\{z \in \mathbf{R}^{\kappa+1}: \inf _{p, q, x}\left\{\left[p^{T} z-q\right]: P[p ; q]-\mathcal{Q} x=0, x \succeq 0\right\} \geq 0\right\} \text { [by[45)] } \\
= & \left\{z \in \mathbf{R}^{\kappa+1}: \exists \lambda \in \mathbf{R}^{2 \kappa+1}: P^{T} \lambda=[z ;-1], \mathcal{Q}^{*} \lambda \succeq 0\right\} \\
& {\left[\text { by semidefinite duality; } \lambda \mapsto \mathcal{Q}^{*} \lambda: \mathbf{R}^{2 \kappa+1} \rightarrow \mathbf{S}^{\kappa+1}\right.} \\
& \text { is the conjugate of } \left.x \mapsto \mathcal{Q} x: \mathbf{S}^{\kappa+1} \rightarrow \mathbf{R}^{2 \kappa+1}\right],
\end{aligned}
$$

and we arrive at the desired SDR of $\mathcal{Z}$.

\section{A.2 Verification of calculus rules}

1. [Restriction on a $\mathcal{K}$-representable set] Let (25) represent $(F, \mathcal{X})$. Suppose that $[t ; g ; x]$ can be augmented by $u, v$ to solve (27). Then $(t, g, x, u)$ solve (25), implying that $[t ; g ; x] \in \mathcal{F}[F, \mathcal{X}]$ by definition of $\mathcal{K}$ representability of $(F, \mathcal{X})$, and $(x, v)$ solve (27), implying that $x \in \mathcal{Y}$. Taking together, these inclusions clearly imply that $[t ; g ; x] \in \mathcal{F}[\bar{F}, \mathcal{Z}]$, as required in item (i) of the definition of $\mathcal{K}$-representation of $(\bar{F}, \mathcal{Z})$. Next, when $x \in \mathcal{Z}$, the triple $[t:=\langle F(x), x\rangle ; g:=F(x) ; x]$ can be augmented by $u$ to solve (25) (by item (ii) of the definition of $\mathcal{K}$-representation of $(F, \mathcal{X})$ ), and because $x \in \mathcal{Y}, x$ can be augmented by $v$ to solve (26). Thus, $t:=\langle F(x), x\rangle, g:=F(x), x$ can be augmented by $(u, v)$ to solve (27), as required in item (ii) of $\mathcal{K}$-representability of $(\bar{F}, Z)$. Thus, (27) indeed represents $(\bar{F}, \mathcal{Z})$. A completely similar reasoning shows that if (25) almost represents $(F, \mathcal{X})$, then (27) almost represents $(\bar{F}, \mathcal{Z})$.

2. [Direct summation] When $x=\left[x_{1} ; \ldots ; x_{K}\right] \in \mathcal{X}$ and $g=F(x)=\left[g_{1} ; \ldots ; g_{K}\right], g_{k}=F_{k}\left(x_{k}\right), t_{k}=\left\langle x_{k}, g_{k}\right\rangle$ and $t=\sum_{k} t_{k}=\langle g, x\rangle$, there exist $u_{k}$ such that [29] $a$ ) take place (by item (ii) of the definition of representation as applied to the representations in (28)), and (29, $b$ ) takes place as well, as required in item (ii) of the definition of a representation of $(F, \mathcal{X})$. On the other hand, when $x=\left[x_{1} ; \ldots ; x_{K}\right]$, $g=\left[g_{1} ; \ldots ; g_{k}\right]$, and $t$ can be augmented by $u_{k}$ and $t_{k}$ to solve $\left.(29) a\right)$ and $(29, b)$, we have $\left[t_{k} ; g_{k} ; x_{k}\right] \in$ $\mathcal{F}\left[F_{k}, \mathcal{X}_{k}\right], k \leq K$, whence for every $y=\left[y_{1} ; \ldots ; y_{K}\right] \in \mathcal{X}$ it holds

$$
t_{k}-\left\langle g_{k}, y_{k}\right\rangle \geq\left\langle F_{k}\left(y_{k}\right), x_{k}-y_{k}\right\rangle, k \leq K .
$$

When summing up the above inequalities over $k$, we get

$$
t-\langle g, y\rangle \geq\langle F(y), x-y\rangle \forall y \in \mathcal{X}
$$

that is, $[t ; g ; x] \in \mathcal{F}[F, \mathcal{X}]$, as required in item (i) of the definition of a representation of $(F, \mathcal{X})$.

The above reasoning, with evident modifications, shows the claim in the case of almost representations.

3. [Taking conic combinations] Let $t, g, x$ can be augmented by $u_{k}, t_{k}, g_{k}, k \leq K$ to solve (31). Then $\left[t_{k} ; g_{k} ; x\right] \in \mathcal{F}\left[\mathcal{F}_{k}, \mathcal{X}\right]$, implying that

$$
t_{k}-\left\langle g_{k}, y\right\rangle \geq\left\langle F_{k}(y), x-y\right\rangle \forall y \in X
$$

multiplying both sides by $\alpha_{k}$ and summing up over $k$, we get

$$
t-\langle g, y\rangle \geq\langle F(y), x-y\rangle \forall y \in X,
$$

that is, $[t ; g ; x] \in \mathcal{F}[F, \mathcal{X}]$. On the other hand, given $x \in \mathcal{X}$, let us set $g_{k}=F_{k}(x)$ and $t_{k}=\left\langle F_{k}(x), x\right\rangle$. Since the $k$-th conic constraint in (31) represents $\left(F_{k}, \mathcal{X}\right)$, there exist $u_{k}, k \leq K$, such that all relations (31 a) take place. Setting $t=\sum_{k} \alpha_{k} t_{k}, g=\sum_{k} \alpha_{k} g_{k}$, we, on one hand, satisfy (31, b-c), and, on the other hand, obtain

$$
t=\sum_{k} \alpha_{k}\left\langle F_{k}(x), x\right\rangle=\langle F(x), x\rangle, g=\sum_{k} \alpha_{k} g_{k}=F(x),
$$

the bottom line being that $[\langle F(x), x\rangle ; F(x) ; x]$ can be augmented by $u_{k}, t_{k}, g_{k}$ to solve (31). Thus, (31) indeed is a $\mathcal{K}$-representation of $(F, \mathcal{X})$.

The above reasoning, with evident modifications, works in the case of almost representations. 
4. [Affine substitution of variables] Assume that $\tau, \gamma, \xi, g, t, u$ solve (33). Then $t, g, x:=A \xi+a, u$ satisfy

$$
X x+G g+t T+U u \leq_{\mathbf{K}} a,
$$

implying that $x \in \mathcal{X}$ and

$$
t-\langle g, y\rangle \geq\langle F(y), x-y\rangle \forall y \in \mathcal{X} .
$$

Recall that $x=A \xi+a, x \in \mathcal{X}$, implies that $\xi \in \Xi$. Now, when $\eta \in \Xi$, setting $y=A \eta+a$, we have $y \in \mathcal{X}$ and

$$
\begin{aligned}
\tau-\langle\gamma, \eta\rangle & =t-\langle g, a\rangle-\left\langle A^{T} g, \eta\right\rangle=t-\langle g, y\rangle \geq\langle F(y), x-y\rangle \\
& =\langle F(A \eta+a), A(\xi-\eta)\rangle=\langle\Phi(\eta), \xi-\eta\rangle,
\end{aligned}
$$

and since $\eta \in \Xi$ is arbitrary, we get $[\tau ; \gamma ; \xi] \in \mathcal{F}[\Phi, \Xi]$, as required in item (i) of the definition of a representation of $(\Phi, \Xi)$. On the other hand, when $\xi \in \Xi, \gamma=\Phi(\xi)$, and $\tau=\langle\Phi(\xi), \xi\rangle$, setting $x=A \xi+a$, we get $x \in \mathcal{X}$. Next, when setting $g=F(x), t=\langle F(x), x\rangle$, we obtain $\gamma=A^{T} g$ and

$$
\tau=\langle F(x), x-a\rangle=t-\langle g, a\rangle .
$$

Besides this, by the origin of $t, g, x$ and item (ii) of the definition of $\mathcal{K}$-representation, as applied to (32), there exists $u$ such that $t, g, x, u$ satisfy (32). The bottom line is that $\xi, \gamma, \tau$ can be augmented by $t, g, u$ to solve (33), that is, (33) meets item (ii) of definition of $\mathcal{K}$-representation of $(\Phi, \Xi)$.

The above reasoning, with evident modifications, works in the case of almost representations.

\section{B Verifying (38) and (39)}

A. Let us prove that for all $u \in \mathcal{U}=[0, U], v \in \mathcal{V}=[0, V]$ one has

$$
\begin{aligned}
\psi(u, v) & =-\frac{u}{u+v+1} \\
& =\min _{f, t, s}\left\{f v+t: 1 \geq f \geq 0,0 \leq s \leq \sqrt{u f}, s+\frac{1}{u+1} \leq 1, t-f \geq(1-s)^{2}-1, t \leq 1\right\} \\
& =\min _{f, t, s ;, \tau}\left\{\begin{array}{c}
1 \geq f \geq 0, s \geq 0, t \leq 1, \tau \geq 0, s+\tau \leq 1 \\
\underbrace{\left[\begin{array}{l}
{[2(1-s) ; t-f ; t-f+2] \in \mathbf{L}^{3}} \\
\Leftrightarrow(1-s)^{2} \leq t-f+1 \text { when } t-f+2 \geq 0 \\
{[2 ; u-\tau+1 ; u+\tau+1] \in \mathbf{L}^{3}}
\end{array}\right.}_{\begin{array}{l}
12 s ; u-f ; u+f] \in \mathbf{L}^{3} \\
\Leftrightarrow s^{2} \leq u f \text { when } u+f \geq 0
\end{array}}
\end{array}\right\} .
\end{aligned}
$$

Indeed, for $u \in \mathcal{U}$ and $v \in \mathcal{V}$ we have

$$
\begin{aligned}
& \min _{f, t, s}\left\{f v+t: 1 \geq f \geq 0,0 \leq s \leq \sqrt{u f}, s+\frac{1}{u+1} \leq 1, t-f \geq(1-s)^{2}-1, t \leq 1\right\} \\
& =\min _{f}\left\{f v+f-2 \bar{s}(f)+\bar{s}(f)^{2}: 1 \geq f \geq 0, \bar{s}(f)=\min \left[\frac{u}{u+1}, \sqrt{u f}\right]\right\} \\
& =\min \left[\min _{0 \leq f \leq u /(u+1)^{2}}\{f v+f-2 \sqrt{u f}+u f\}, \min _{1 \geq f \geq u /(u+1)^{2}}\left\{f v+f-\frac{2 u}{u+1}+\frac{u^{2}}{(u+1)^{2}}\right\}\right] \\
& =\min \left[-\frac{u}{u+v+1}, \frac{u(v+1)-2 u-u^{2}}{(u+1)^{2}}\right]=u \min \left[-\frac{1}{v+(u+1)}, \frac{v-(u+1)}{(u+1)^{2}}\right]=\frac{u}{v+u+1} \min \left[-1, \frac{v^{2}-(u+1)^{2}}{(u+1)^{2}}\right] \\
& =\psi(u, v),
\end{aligned}
$$

as claimed. 
B. Now let us show that for all $u \in \mathcal{U}=[0, U], v \in \mathcal{V}=[0, V]$

$$
\begin{aligned}
& -\psi(u, v):=\frac{u}{u+v+1} \\
& =\min _{f, t, s}\left\{f u+t: 1 \geq f \geq 0,0 \leq s \leq \sqrt{(v+1) f}, s \leq 1,1 \geq t \geq(1-s)^{2}\right\}
\end{aligned}
$$

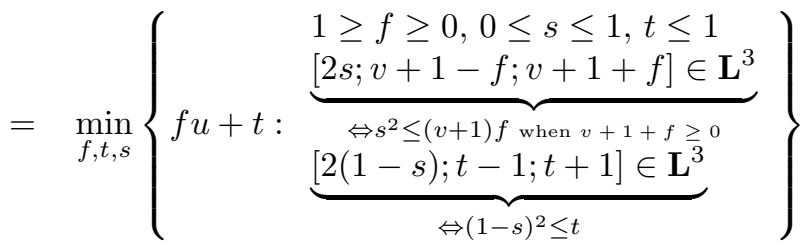

Indeed, for $u \in \mathcal{U}$ and $v \in \mathcal{Q}$ we have

$$
\begin{aligned}
& \min _{f, t, s}\left\{f u+t: 1 \geq f \geq 0,0 \leq s \leq \sqrt{(v+1) f}, s \leq 1,1 \geq t \geq(1-s)^{2}\right\} \\
& =\min _{f, s}\left\{f u+(1-s)^{2}: 1 \geq f \geq 0,0 \leq s \leq \sqrt{(v+1) f}, s \leq 1\right\} \\
& =\min _{f}\left\{f u+(1-\bar{s}(f))^{2}: 1 \geq f \geq 0, \bar{s}(f)=\min [\sqrt{(v+1) f}, 1]\right\} \\
& =\min \left[\min _{0 \leq f \leq 1 /(v+1)}\{f u+1-2 \sqrt{(v+1) f}+(v+1) f\}, \min _{1 \geq f \geq 1 /(v+1)}\{f u\}\right] \\
& =\min \left[\frac{u}{u+v+1}, \frac{u}{v+1}\right]=\frac{u}{u+v+1}=-\psi(u, v),
\end{aligned}
$$

as claimed. 